\title{
Non-Self-Dual Yang-Mills Connections with Quadrupole Symmetry
}

\author{
Lorenzo Sadun ${ }^{1, \star}$ and Jan Segert ${ }^{2, \star \star}$ \\ 1 Courant Institute of Mathematical Sciences, New York University, 251 Mercer Street, New York, \\ NY 10012, USA \\ 2 Department of Mathematics, Mathematical Sciences Building, University of Missouri, Columbia, \\ MO 65211, USA
}

Received September 30, 1991

\begin{abstract}
We prove the existence of non-self-dual Yang-Mills connections on $S U(2)$ bundles over the four-sphere, specifically on all bundles with second Chern number not equal \pm 1 . We study connections equivariant under an $S U(2)$ symmetry group to reduce the effective dimensionality from four to one, and then use variational techniques. The existence of non-self-dual $S U(2)$ YM connections on the trivial bundle (second Chern number equals zero) has already been established by Sibner, Sibner, and Uhlenbeck via different methods.
\end{abstract}

\section{Introduction}

\subsection{Background and Statement of the Main Result}

In this paper we prove the existence of non-self-dual Yang-Mills connections on topologically nontrivial $S U(2)$ bundles over the four-sphere $S^{4}$, with the standard metric. For brevity, we use the term non-self-dual to refer to connections that are neither self-dual nor anti-self-dual. Recall that the $S U(2)$ bundles over $S^{4}$ are topologically classified by $C_{2}$, the second Chern number of the associated vector bundles. The existence of non-self-dual Yang-Mills connections on the trivial $S U(2)$ bundle over $S^{4}$ has been proven by Sibner, Sibner, and Uhlenbeck [SSU]. As announced in [SS2], we prove that non-self-dual YM connections exist on all $S U(2)$ bundles with $C_{2} \neq \pm 1$. Existence for $C_{2}= \pm 1$ is still an open problem.

The study of non-self-dual Yang-Mills connections has developed rather slowly compared to the progress made in the understanding of self-dual connections. The first nontrivial solution of the Yang-Mills equations on $S^{4}$ was the self-dual $S U(2)$ instanton, found in 1975 [BPST]. Three years later, the self-dual solutions on $S^{4}$ were

\footnotetext{
* Research partially supported by NSF Grant DMS-8806731

Present address: Mathematics Department, University of Texas, Austin, TX 78712, USA

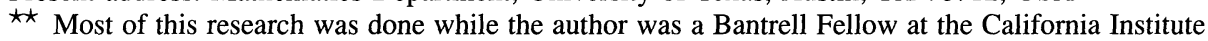
of Technology, and was partially supported by NSF Grant DMS- 8801918
} 
classified [ADHM], not just for $S U(2)$ but for all classical groups. The study of selfdual $S U(2)$ connections on four-manifolds led to spectacular progress in topology, notably in the works of Donaldson (see [FU] for an overview).

In contrast, the understanding of non-self-dual YM connections is at present relatively modest, falling far short of a classification theory. In 1981, Itoh [I] found non-self-dual YM connections on $S^{4}$ with structure groups $S U(k), k \geq 4$, and other large groups. Itoh's YM connections are equivariant under the full $S O(5)$ symmetry group. In 1984 Manin used twistor methods to find other examples of non-self-dual YM connections on $S^{4}$ which had rather complicated gauge groups (and supergroups) [Ma1; Ma2, Sects. 2.7, 2.9, 5.5]. Urakawa [Ur] found non-self-dual YM connections on $S U(2)$ bundles over $S^{2} \times S^{2}$ and over $S^{3} \times S^{1}$. Parker [P1, P2] has recently proven the existence of non-self-dual $S U(2)$ YM connections on $S^{3} \times S_{L}^{1}$, where $S_{L}^{1}$ is a circle of circumference $L$, and on $S_{g}^{4}$, the four-sphere with a nonstandard metric.

Non-self-dual YM connections on $S U(2)$ bundles over the standard four-sphere proved elusive, however, and there were reasons to believe that they might not exist. The $S U(2)$ Yang-Mills connections on $S^{4}$ are formally analogous to harmonic maps from the Riemann sphere to itself [AJ]. This analogy seemed to suggest that all $S U(2)$ YM connections on $S^{4}$ should be (anti)self-dual. Bourguignon and Lawson [BL] (see also [T1, BLS]) showed that every weakly stable ${ }^{1} S U(2)$ Yang-Mills connection on $S^{4}$ is self-dual (or anti-self-dual). Taubes [T2, JT] showed that all $S U(2)$ YM connections that are equivariant with respect to a certain $O(3)$ action are (anti)self-dual. Despite these apparent indications of nonexistence, Sibner, Sibner, and Uhlenbeck have recently demonstrated that there exist infinitely many non-self-dual $S U(2)$ YM connections on the topologically trivial bundle over the four-sphere [SSU].

The basic technique of this paper is to consider solutions with a symmetry group action that reduces the dimensionality of the Yang-Mills equations and the self-duality equations from four to one. The general formalism of dimensional reduction of the Yang-Mills equations from four dimensions to one has been developed by Urukawa [Ur], and has been worked out in this specific context by Bor and Montgomery [BoMo]. Sibner, Sibner, and Uhlenbeck [SSU] used quite different techniques, namely minmax theory for monopoles on hyperbolic space.

The symmetry group we consider is $S U(2)$, and the action on $S^{4}$ is that induced from the unique irreducible representation of $S U(2)$ on $\mathbf{R}^{5}$. The inequivalent lifts of this action to $S U(2)$ bundles over $S^{4}$ are naturally classified by a pair of odd positive integers $\left(n_{+}, n_{-}\right)$. We shall refer to each such bundles $P_{\left(n_{+}, n_{-}\right)} \rightarrow S^{4}$ as a quadrupole bundle; these bundles appeared in [ASSS] as spectral bundles of quantum-mechanical quadrupole operators. Our main result is:

Theorem 1.1. On each quadrupole bundle $P_{\left(n_{+}, n_{-}\right)} \rightarrow S^{4}$ with $n_{+} \neq 1$ and $n_{-} \neq 1$, there exists a smooth non-self-dual SU(2) Yang-Mills connection. These connections are all distinct.

On the bundles with $n_{+}=1$ or $n_{-}=1$, we believe that there exist (anti)selfdual equivariant connections, although we do not prove this in the present paper. We remark that the flat connection is equivariant on $P_{(1,1)}$, and the BPST instanton is equivariant on $P_{(3,1)}$, see [ASSS]. Recently Bor $^{2}$ has established the existence of a self-dual connection on $P_{(5,1)}$ via an equivariant ADHM construction.

\footnotetext{
1 A YM connection is weakly stable if the second variation is nonnegative

2 Gil Bor, private communication
} 
The second Chern number of a quadrupole bundle $P_{\left(n_{+}, n_{-}\right)}$is given by $C_{2}=$ $\left(n_{+}^{2}-n_{-}^{2}\right) / 8$ ([SS1, ASSS]). From this and Theorem 1.1 above, one can easily deduce the following facts. Non-self-dual Yang-Mills connections exist on every topological bundle type with $C^{2} \neq \pm 1$. In fact, for a bundle with $C_{2}=k$, the number of distinct non-self-dual YM connections we obtain is equal to the number of distinct odd positive factors of $k$ (not necessarily prime), minus one if $k= \pm n(n+1) / 2$ for some $n=1,2,3 \ldots$ For example, $k=-45$ has six positive odd factors, 1, 3, 9, $5,15,45$, and furthermore $-45=-9(9+1) / 2$, so the number of distinct solutions we obtain is five. For the trivial bundle $k=0$, we obtain a countably infinite set of distinct solutions, which are of different symmetry type than the solutions of [SSU].

\subsection{Outline of the Paper}

In Sect. 2 we discuss the $S U(2)$ action on the quadrupole bundles, and connections that are equivariant under this action. Much of this material is due to Urakawa [Ur] and to Bor and Montgomery [BoMo]. We denote by $X \subset S^{4}$ the dense open submanifold consisting of the principal (three-dimensional) orbits of the symmetry group. The Yang-Mills and self-duality equations on $X$ are reduced to one dimension, as systems of ODE's on the interval $(0, \pi / 3)$.

We label the equivariant connections on $X$ with two real numbers, $r$ and $t$, which describe holonomy near the singular orbits of the symmetry group in $S^{4}$. If $r=$ $-1(\bmod 4)$ and $t=-1(\bmod 4)$, then a finite-action connection on $X \subset S^{4}$ extends to a connection on the quadrupole bundle $P_{\left(n_{+}, n_{-}\right)} \rightarrow S^{4}$, where $n_{+}=|r|$ and $n_{-}=|t|$. However, if $r \neq-1(\bmod 4)$ or $t \neq-1(\bmod 4)$, there is a holonomy obstruction to extending the connection, and the connection has "fractional Chern number" [FHP1, FHP2, SiSi1, SiSi2] equal to $\left(r^{2}-t^{2}\right) / 8$.

In Sect. 3 we solve the one-dimensional problem, showing that non-self-dual YangMills solutions exist on $X$ for all $(r, t)$ with $|r|>1,|t|>1$ (Theorem 3.1). The existence of Yang-Mills solutions (Theorem 3.10) is proved with the direct method in the calculus of variations. The usual difficulties caused by conformal invariance (bubbling off [SaU, Uh1, Uh3]) and gauge invariance do not occur, because we are working in one dimension. The nonexistence of (anti)self-dual solutions (Theorem 3.11) comes from analyzing the boundary values of solutions of the one dimensional (anti)self-dual equations.

In Sect. 4 we show that the non-self-dual YM solutions on $X$ extend to smooth solutions on quadrupole bundles $P_{\left(n_{+}, n_{-}\right)} \rightarrow S^{4}$ for the appropriate values of $(r, t)$ (Theorem 4.1). This is a straightforward application of Uhlenbeck's theorem [Uh2] on the existence of a gauge choice (Coulomb gauge), which together with the YangMills equations forms a uniformly elliptic system of PDE's. Most of this chapter consists of verifying that in a certain gauge, our connections belong to the appropriate Sobolev space. Uhlenbeck's theorem then gives the existence of a Sobolev gauge transformation which takes our gauge to Coulomb gauge, and the smoothness follows by eliptic regularity theory. The symmetry of our connection actually forces the gauge transformation to be continuously differentiable, eliminating the difficulties associated with noncontinuous Sobolev gauge transformations changing the topological type of the bundle. ${ }^{3}$

$\overline{3}$ There are similarities here with the equivariant Sobolev theorems announced by Parker [P1] 


\subsection{Numerical Approximations}

Having established the existence of equivariant non-self-dual Yang-Mills connections, it remains to consider the form of the solutions and the geometry of the various moduli spaces. While we have not yet found any closed-form expressions for these solutions, we have numerically computed the connection and the minimizing action for several values of $\left(n_{+}, n_{-}\right)$. For example, on $P_{(3,3)}$ the solution has action $5.432815071 \pm$ 0.00000001 times the action of the BPST instanton. We find that the action grows rapidly with $n_{ \pm}$, and that distinct solutions with the same Chern number do not have the same action, which led us to conjecture in [SS2] that non-self-dual YM moduli space is disconnected. The numerical work will appear in [SS3].

\section{The Symmetry Group Action}

In this chaper we discuss the $S U(2)$ group action on the quadrupole bundles $P_{\left(n_{+}, n_{-}\right)}$ $\rightarrow S^{4}$. The equivariant connections are described by triplets of real functions on an interval $(0, \pi / 3)$, and the YM equations and (anti)self-dual equations reduce to systems of ordinary differential equations.

\subsection{The Symmetry of the Four-Sphere}

We will consider the symmetry group $G=S U(2)=\mathrm{Sp}(1)$. Recall that $\mathrm{Sp}(1)$ is the unit sphere in the quaternions $\mathbf{H}$. Let $\left(1, \varrho_{1}, \varrho_{2}, \varrho_{3}\right)$ be a basis of $\mathbf{H}$, with

$$
\varrho_{i} \varrho_{i}=-1, \quad \varrho_{1} \varrho_{2}=-\varrho_{2} \varrho_{1}=\varrho_{3}, \quad \text { etc. }
$$

For $h \in G$, let $R_{h}: G \rightarrow G$ be the right translation taking $g \mapsto g h$. Similarly the left translation $L_{h}$ takes $g \mapsto h g$.

The Lie algebra $\operatorname{Lie}(G)$ is the space of left-invariant vector fields on $G$; a vector field $l \in \operatorname{Lie}(G)$ satisfies $\left(L_{h}\right)_{*} l=l$ for any $h \in G$. $\operatorname{Lie}(G)$ inherits the algebraic structure from the commutator operation on vector fields. Let $h(t), t \in \mathbf{R}$ be a oneparameter subgroup of $G$. Then the vector field

$$
\left.\frac{d}{d t} R_{h(t)} g\right|_{t=0} \in T_{g} G
$$

is left-invariant (since left translations commute with right translations). Take as a basis of $\operatorname{Lie}(G)$ the vector fields $l_{i}, i=1,2,3$, that correspond to the right translations by $\exp \left(t \varrho_{i} / 2\right)$. Then $\left[l_{1}, l_{2}\right]=l_{3}$ and (cyclic), by which we mean that the equation holds not only for the indices $1,2,3$, but as well for the cyclic permutations $2,3,1$ and $3,1,2$. We will often use the fact that $G$ is a subgroup of a linear group to identify $\exp \left(t l_{i}\right)=\exp \left(t \varrho_{i} / 2\right)$. Denoting by $\beta^{i}$ the basis of left-invariant one-forms dual to $l_{i}$, the Maurer-Cartan equations are $d \beta^{1}=-\beta^{2} \wedge \beta^{3}$, and (cyclic).

The irreducible representation of $G$ on $V \cong \mathbf{R}^{5}$ factors through a representation of $S O(3)$, which we now construct. Let $V$ be the 5 dimensional vector space of real traceless symmetric $3 \times 3$ matrices, with norm $\|Q\|^{2}=\frac{1}{2} \operatorname{Tr}\left(Q^{2}\right)$. The linear action

$$
\begin{aligned}
S O(3) \times V & \rightarrow V \\
(g, Q) & \mapsto g Q g^{-1}
\end{aligned}
$$


defines a norm-preserving representation $c: S O(3) \rightarrow \operatorname{Aut}(V)$. Composing with the double cover $j: S U(2) \rightarrow S O(3)$, we obtain a representation $b=c \circ j: G \rightarrow \operatorname{Aut}(V)$.

It is convenient to have explicity matrix bases. Choose a basis of $\mathbf{R}^{3}$ such that the matrices $K_{i} \equiv j_{*} l_{i} \in \operatorname{Lie}(S O(3))$ are

$$
K_{1}=\left(\begin{array}{ccc}
0 & 0 & 0 \\
0 & 0 & -1 \\
0 & 1 & 0
\end{array}\right), \quad K_{2}=\left(\begin{array}{ccc}
0 & 0 & 1 \\
0 & 0 & 0 \\
-1 & 0 & 0
\end{array}\right), \quad K_{3}=\left(\begin{array}{ccc}
0 & -1 & 0 \\
1 & 0 & 0 \\
0 & 0 & 0
\end{array}\right) .
$$

A basis for the representation $b_{*}: \operatorname{Lie}(G) \rightarrow \operatorname{End}(V)$ is then given by

$$
\begin{aligned}
R_{i} \equiv b_{*} l_{i}: V & \rightarrow V \\
Q & \mapsto\left[K_{\imath}, Q\right],
\end{aligned}
$$

where of course $\left[R_{1}, R_{2}\right]=R_{3}$, and (cyclic). An orthonormal basis of $V$ is given by

$$
\begin{gathered}
Q_{0}=\frac{1}{\sqrt{3}}\left(\begin{array}{ccc}
-1 & 0 & 0 \\
0 & -1 & 0 \\
0 & 0 & 2
\end{array}\right), \quad Q_{1}=\left(\begin{array}{lll}
0 & 0 & 1 \\
0 & 0 & 0 \\
1 & 0 & 0
\end{array}\right), \\
Q_{2}=\left(\begin{array}{lll}
0 & 0 & 0 \\
0 & 0 & 1 \\
0 & 1 & 0
\end{array}\right), \quad Q_{3}=\left(\begin{array}{ccc}
1 & 0 & 0 \\
0 & -1 & 0 \\
0 & 0 & 0
\end{array}\right), \quad Q_{4}=\left(\begin{array}{lll}
0 & 1 & 0 \\
1 & 0 & 0 \\
0 & 0 & 0
\end{array}\right) .
\end{gathered}
$$

The restriction of the representation to the unit sphere in $S^{4} \subset V$ induces an isometric action of $G$ on $S^{4}$, which we now study via the matrix realization above. Let $I=(0, \pi / 3) \subset \mathbf{R}$, and $\bar{I}=[0, \pi / 3]$. The path $Q_{\theta} \equiv \cos (\theta) Q_{0}+\sin (\theta) Q_{3}$ is a geodesic on $S^{4}$, with affine parameter $\theta \in \mathbf{R}$. Points on this geodesic are diagonal matrices in $V$. We now define some subgroups of $G=\operatorname{Sp}(1)$ : let $\Gamma=\left\{ \pm 1, \pm \varrho_{1}, \pm \varrho_{2}, \pm \varrho_{3}\right\}$ and, for $i=1,2,3$, let $L_{i}=\left\{\exp \left(\phi \varrho_{i}\right) ; \phi \in[0,2 \pi)\right\}$.

Lemma 2.1. There is a closed geodesic segment of length $\pi / 3$ on $S^{4}$ which intersects each $G$-orbit exactly once. The principal orbits are three dimensional, and there are two exceptional orbits of dimension two.

Proof. Every matrix in $S^{4} \subset V$ is diagonalizable, and so is related by conjugation to some diagonal matrix $Q_{\theta}$. The rotation $\exp \left(\pi K_{3} / 2\right)$ maps $Q_{\theta} \rightarrow Q_{-\theta}$, and the rotations $\exp \left( \pm 2 \pi\left(K_{1}+K_{2}+K_{3}\right) / 3 \sqrt{3}\right.$ map $Q_{\theta} \rightarrow Q_{\theta \mp 2 \pi / 3}$, so we can restrict out attention to $\theta \in \bar{I}$. Since the function $\operatorname{Det}\left(Q_{\theta}\right)=\frac{2}{3 \sqrt{3}} \cos (3 \theta)$ is $1-1$ on $\bar{I}$, the reduction can go no further. The geodesic segment $Q_{\theta}, \theta \in \bar{I}$, thus intersects each $S O(3)$ orbit exactly once, and so intersects each $G$ orbit exactly once.

For $\theta \in I$, the subgroup of $G$ that leaves $Q_{\theta}$ fixed is $\Gamma$, which is finite, so the orbit $G / \Gamma$ is three dimensional. $Q_{0}$ is fixed by the group $J_{0}$ which is generated by the subgroups $\Gamma$ and $L_{3} . L_{3}$ is a one-parameter group, so the orbit of $Q_{0}$ is twodimensional. Similarly, $Q_{\pi / 3}$ is fixed by the group $J_{\pi / 3}$, which is generated by $\Gamma$ and $L_{2}$.

Let $X \subset S^{4}$ be the union of the three-dimensional orbits. $X$ is a dense open submanifold of $S^{4}$. It is convenient to consider a covering space of $X$ on which $G$ acts freely. Define $Y \equiv I \times G$, with a right action of $\Gamma \subset G$ by $G$-translation on the second factor. Then $Y / \Gamma \cong X$, and the projection map is

$$
\begin{aligned}
s: Y & \rightarrow X \\
(\theta, g) & \rightarrow g Q_{\theta},
\end{aligned}
$$

where $g$ denotes the action (2.1) of $g \in G$ on $X \subset S^{4}$. 
The topological classification of bundles over $Y=I \times G$ coincides with that over $G \cong S^{3}$, since these spaces are homotopic. $K$-bundles over $S^{3}$ are classified by $\pi_{2}(K)$, which is trivial for all semisimple Lie groups. The tangent bundle $T Y$ is thus topologically trivial, and admits a global basis of vector fields. One such basis is given by the vector fields $\left\{\partial / \partial \theta, l_{1}, l_{2}, l_{3}\right\}$.

Let $g$ be the canonical Riemannian metric on $S^{4}$, induced from the invariant inner product on $V$. The pullback metric $s^{*} g$ on $Y$ is invariant under left translation on the second factor. Defining

$$
f_{1}(\theta)=2 \sin (\pi / 3+\theta) ; \quad f_{2}(\theta)=2 \sin (\pi / 3-\theta) ; \quad f_{3}(\theta)=2 \sin (\pi),
$$

we have:

Lemma 2.2. The one-forms

$$
\alpha^{0} \equiv d \theta ; \quad \alpha^{i} \equiv f_{i} \beta^{i}, \quad i=1,2,3
$$

on $Y=I \times G$ are invariant under left translation on the second factor, and constitute an orthonormal basis of the cotangent bundle $T^{*} Y$.

Proof. The invariance is immediate. Orthonormality is by direct calculation. We show that the dual basis

$$
e_{0} \equiv \frac{\partial}{\partial \theta} ; \quad e_{i} \equiv \frac{l_{i}}{f_{i}}, \quad i=1,2,3
$$

of the tangent bundle $T Y$ is orthonormal at $(\theta, 1) \in Y$ by checking that the vectors $s_{*} e_{i}$ are orthonormal at $Q_{\theta} \in S^{4}$.

Using the explicit matrix bases, we compute in $T_{Q_{\theta}} S^{4}$

$$
\begin{aligned}
s_{*} l_{1} & =R_{1}\left(Q_{\theta}\right)=\cos (\theta)\left[K_{1}, Q_{0}\right]+\sin (\theta)\left[K_{1}, Q_{3}\right], \\
& =-f_{1}(\theta) Q_{2} \\
s_{*} l_{2} & =R_{2} Q_{\theta}=f_{2}(\theta) Q_{1} \\
s_{*} l_{3} & =R_{3} Q_{\theta}=f_{3}(\theta) Q_{4} \\
s_{*} \frac{\partial}{\partial \theta} & =d Q_{\theta} / d \theta=-\sin (\theta) Q_{0}+\cos (\theta) Q_{3}
\end{aligned}
$$

where the linear maps $R_{i}$ are as in (2.2). Since $Q_{0}, \ldots, Q_{4}$ are orthonormal in $V$, the orthonormality of the $e_{i}$ 's follows.

The Riemannian volume form on $Y$, relative to the metric $s^{*} g$, is given by

$$
\eta=\alpha^{0} \wedge \alpha^{1} \wedge \alpha^{2} \wedge \alpha^{3}=f_{1} f_{2} f_{3} d \theta \wedge \beta^{1} \wedge \beta^{2} \wedge \beta^{3}
$$

and we compute the Hodge dual on a basis of two-forms:

$$
*\left(d \theta \wedge \beta^{1}\right)=G_{1} \beta^{2} \wedge \beta^{3} ; \quad *\left(d \theta \wedge \beta^{2}\right)=G_{2} \beta^{3} \wedge \beta^{1} ; \quad *\left(d \theta \wedge \beta^{3}\right)=G_{3} \beta^{3} \wedge \beta^{2},
$$

where

$$
G_{1} \equiv \frac{f_{2} f_{3}}{f_{1}} ; \quad G_{2} \equiv \frac{f_{3} f_{1}}{f_{2}} ; \quad G_{3} \equiv \frac{f_{1} f_{2}}{f_{3}}
$$




\subsection{Quadrupole Bundles}

The quadrupole bundles of [ASSS] are a family of $S U(2)$ bundles over $S^{4}$ together with an action of $G$ by bundle morphisms. The $G$-action induced on the base space $S^{4}$ coincides with the action that comes from the irreducible representation of $G$ on $V=\mathbf{R}^{5}$. The bundles arose as the spectral projections of certain families of quaternionic operators [ASSS], but our discussion here follows the description of Bor and Montgomery [BoMo] and Urakawa [Ur].

For every pair $\left(n_{+}, n_{-}\right)$of positive odd integers there exists a distinct quadrupole bundle. ${ }^{4}$ Each bundle is distinct in the sense that no two quadrupole bundles are isomorphic via a bundle map that commutes with the $G$-actions. However, restricted to $X \subset S^{4}$ all the quadrupole bundles are isomorphic to a bundle $P_{X} \rightarrow X$. In this section, we first describe $P_{X} \rightarrow X$ and then construct the extensions to the quadrupole bundles $P_{\left(n_{+}, n_{-}\right)} \rightarrow S^{4}$.

Let $H=S U(2)=\mathrm{Sp}(1)$ and let $P_{Y}=Y \times H$ be the trivial principal bundle. Then $H$ acts on the right,

$$
(\theta, g, h) \mapsto\left(\theta, g, h h^{\prime}\right), \quad h^{\prime} \in H .
$$

Let $G$ act on $P_{Y}$ by

$$
(\theta, g, h) \mapsto\left(\theta, g^{\prime} g, h\right), \quad g^{\prime} \in G .
$$

This left $G$ action commutes with the $H$ action (2.9), i.e., $G$ acts by bundle morphisms. by

We now introduce an equivalence relation $\sim$ on $P_{Y}$. Let $\Gamma$ act on $P_{Y}$ on the right

$$
(\theta, g, h) \mapsto\left(\theta, g \gamma, \gamma^{-1} h\right), \quad \gamma \in \Gamma,
$$

where we identify $H=G=\operatorname{Sp}(1)$. Then $p \sim q \in P_{y}$ if $p=q \gamma$ for some $\gamma \in \Gamma$, i.e.,

$$
(\theta, g, h) \sim\left(\theta, g \gamma, \gamma^{-1} h\right), \quad \gamma \in \Gamma \text {. }
$$

The $\Gamma$ action commutes with the $H$ action (2.9), and the resulting right action of $H \times \Gamma$ is free. The quotient $P_{X} \equiv P_{Y} / \sim$ is a principal $H$ bundle, with base space $X=Y / \Gamma$. The $G$ action (2.10) also commutes with the $\Gamma$ action, passing to a $G$ action by bundle morphisms on $P_{X}$. The action induced on the base space $X \subset S^{4}$ coincides with the action induced by the irreducible representation of $G$ on $\mathbf{R}^{5}$.

Let $K$ be the canonical section of the trivial bundle $P_{Y}=Y \times H$ :

$$
\begin{aligned}
\kappa: Y & \rightarrow Y \times H \\
(\theta, g) & \mapsto(\theta, g, 1) .
\end{aligned}
$$

$\kappa$ is $G$-equivariant, in that $\kappa \circ g=g \circ \kappa$ where in the first instance, $g$ is the action on $Y$, and in the second instance, on $P_{Y}$.

Now we construct the quadrupole bundles $P_{\left(n_{+}, n_{-}\right)} \rightarrow S^{4}$. Let $\bar{Y} \equiv \bar{I} \times G$, and consider the trivial bundle $P_{\bar{Y}} \equiv \bar{Y} \times H$, with $H$ acting on the right by (2.9) and $G$ acting on the left by (2.10). $\bar{Y}$ maps onto $S^{4}$ by the obvious extension of the maps $s$ in Eq. (2.4). Recall that the isotropy group $J_{\theta}$ of $Q_{\theta}$ for $\theta \in I$ is the group $\Gamma$, that the isotropy group $J_{0}$ of $Q_{0}$ is generated by $\Gamma$ and $L_{3}$, and the isotropy group $J_{\pi / 3}$ of $Q_{\pi / 3}$ is generated by $\Gamma$ and $L_{2}$.

\footnotetext{
${ }^{4}$ Comparing notation with [ASSS], $n_{+}=2\left|m_{T}\right|$, and $n_{-}=2\left|m_{B}\right|$. Comparing with [BoMo], our $n_{ \pm}$are the absolute values of their $n_{ \pm}$
} 
We extend the equivalence relation $\sim$. Let $r$ and $t$ be integers with $r=-1(\bmod 4)$ and $t=-1(\bmod 4)$, and define $n_{+}=|r|$ and $n_{-}=|t|$. For $\theta \in I$, define the homomorphism

$$
\begin{aligned}
\lambda_{\theta}: J_{\theta} & \rightarrow H \\
\gamma & \mapsto . \gamma
\end{aligned}
$$

For $\theta=0$, let

$$
\begin{aligned}
\lambda_{0}: J_{0} & \mapsto H \\
\gamma & \mapsto \gamma, \quad \gamma \in \Gamma \\
\exp \left(\phi \varrho_{3}\right) & \mapsto \exp \left(-r \phi \varrho_{3}\right) .
\end{aligned}
$$

Since $\varrho_{3}$ belongs to both $\Gamma$ and $L_{3}$, the condition $r=-1(\bmod 4)$ is both necessary and sufficient for the consistency of this definition. Similarly for $\theta=\pi / 3$,

$$
\begin{aligned}
\lambda_{\pi / 3}: J_{\pi / 3} & \rightarrow H \\
\gamma & \mapsto \gamma, \quad \gamma \in \Gamma \\
\exp \left(\phi \varrho_{2}\right) & \mapsto \exp \left(-t \phi \varrho_{2}\right) .
\end{aligned}
$$

We define $P_{\left(n_{+}, n_{-}\right)}$to be the quotient of $P_{\bar{Y}}$ under the equivalence relation

$$
(\theta, g, h) \sim\left(\theta, g j, \lambda_{\theta}\left(j^{-1}\right) h\right), \quad j \in J_{\theta} .
$$

The restriction of the equivalence relation to $P_{Y} \subset P_{\bar{Y}}$ coincides with (2.12), so the restriction of $P_{\left(n_{+}, n_{-}\right)} \rightarrow S^{4}$ to $X \subset S^{4}$ is $P_{X}$.

The local product structure of $P_{\left(n_{+}, n_{-}\right)}$over $X$ is already clear. Following [Ur, Sect. 1.3], we now describe the local product structure of $P_{\left(n_{+}, n_{-}\right)}$over a neighborhood $U$ of $Q_{0}$ in $S^{4}$, by constructing a local section. The local product structure over a neighborhood of $Q_{\pi / 3}$ is completely analogous.

We first specify the neighborhood $U$. Let $D$ be the open disk

$$
D=\left\{\left(y_{1}, y_{2}\right) \in \mathbf{R}^{2} \mid y_{1}^{2}+y_{2}^{2}<(\pi / 4)^{2}\right\},
$$

and let $S=\mathbf{R}(\bmod 2 \pi)$ be a cricle. The map

$$
\begin{aligned}
\psi: D \times S & \rightarrow G \\
\left(y_{1}, y_{2}, y_{3}\right) & \mapsto \exp \left(y_{1} \varrho_{1}+y_{2} \varrho_{2}\right) \exp \left(y_{3} \varrho_{3}\right)
\end{aligned}
$$

is a diffeomorphism ${ }^{5}$ of $D \times S$ onto its image $N \equiv \psi(D \times S) \subset G . N$ is clearly invariant under right translations by $L_{3}$. Since $L_{3}$ and $\varrho_{1}$ generate $J_{0}$, the union $M \equiv N \cup N \varrho_{1}$ is $J_{0}$-invariant, and furthermore $N \cap N \varrho_{1}=\emptyset$. Letting $\bar{E}=[0, \pi / 6]$, the quotient of $\bar{E} \times M \subset \bar{Y}$ by the equivalence relation defines the neighborhood $U \subset S^{4}$.

To construct a section, we first define a map $\varphi: M \rightarrow H$. For $g \in N$

$$
\varphi(g) \equiv \exp \left(r y_{3} \varrho_{3}\right) \in H .
$$

Every $g^{\prime} \in N \varrho_{1}$ is uniquely expressed as $g^{\prime}=g \varrho_{1}$ for some $g \in N$, and we define

$$
\varphi\left(g^{\prime}\right)=\varrho_{1}^{-1} \varphi(g)
$$

\footnotetext{
5 Although the function $y_{3}$ is defined on $S$ only modulo $2 \pi$, the map $\psi$ is well-defined, since $\exp \left(2 \pi \varrho_{3}\right)=1$. Similarly, the one-form $d y_{3}$ (by abuse of notation) is well-defined and closed, though not exact
} 
where $\varphi(g)$ is given by (2.15). Note that $\varphi$ cannot be continuously extended from $M$ to $G$.

Denoting by $P_{\bar{E} \times M}$ the restriction of the bundle $P_{\bar{Y}}$ to $\bar{E} \times M \subset \bar{Y}$, we have

Lemma 2.3. The section

$$
\begin{aligned}
\delta: \bar{E} \times M & \rightarrow P_{\bar{E} \times M} \\
(\theta, g) & \mapsto(\theta, g, \varphi(g))
\end{aligned}
$$

passes to the quotient, giving a local section of $P_{\left(n_{+}, n_{-}\right)}$over the neighborhood $U$ of $Q_{0}$ in $S^{4}$.

Proof. Using Urakawa's general results [Ur, Sect. 1.3], we only need to prove that the map $\varphi: M \rightarrow H$ satisfies

$$
\varphi(g j)=\lambda_{0}\left(j^{-1}\right) \varphi(g), \quad g \in M, j \in J_{0} .
$$

For $j=\varrho_{1}$, the proof of (2.18) is immediate from Eq. (2.16). For $j=\exp \left(\phi \varrho_{3}\right) \in$ $L_{3} \subset J_{0}$ and $g \in N$, we compute

$$
\begin{aligned}
\psi(g j) & =\varphi\left(\exp \left(y_{1} \varrho_{1}+y_{2} \varrho_{2}\right) \exp \left(y_{3} \varrho_{3}\right) \exp \left(\phi \varrho_{3}\right)\right. \\
& =\varphi\left(\exp \left(y_{1} \varrho_{1}+y_{2} \varrho_{2}\right) \exp \left(\left(y_{3}+\phi\right) \varrho_{3}\right)\right) \\
& =\exp \left(r\left(y_{3}+\phi\right) \varrho_{3}\right) \\
& =\exp \left(r \phi \varrho_{3}\right) \exp \left(r y_{3} \varrho_{3}\right) \\
& =\lambda_{0}\left(j^{-1}\right) \varphi(g) .
\end{aligned}
$$

Since $L_{3}$ and $\varrho_{1}$ generate $J_{0}$, Eq. (2.18) holds.

The section $\delta$ defines the local product structure of $P_{\left(n_{+}, n_{-}\right)}$.

\subsection{Equivariant Connections}

A connection $\tilde{\omega}$ on $P_{X}$ corresponds to a $\Gamma$-equivariant ${ }^{6}$ connection $\omega=s^{*} \tilde{\omega}$ on $P_{Y}$, since $P_{X}=P_{Y} / \Gamma$. In particular, a $G$-equivariant connection $\tilde{\omega}$ on $P_{X}$ corresponds to a $G$-equivariant and $\Gamma$-equivariant connection $\omega$ on $P_{Y}$.

Let $A_{\kappa} \equiv \kappa^{*} \omega$ be the connection form relative to the section $\kappa . A_{\kappa}$ is a $\operatorname{Lie}(H)$ valued one-form on $Y . A_{\kappa}$ is $G$-invariant, since $\kappa$ is $G$-equivariant, and the most general invariant expression is

$$
A_{\kappa}=\beta^{1} \otimes A_{1}+\beta^{2} \otimes A_{2}+\beta^{3} \otimes A_{3}+d \theta \otimes A_{0},
$$

where the $A_{i}$ are $\operatorname{Lie}(H)$ valued functions of $\theta \in I$. However, $A_{\kappa}$ is not in general invariant under the action (2.11) of $\Gamma$. Requiring $\Gamma$ invariance further restricts this expression.

We call a triplet $a=\left(a_{1}, a_{2}, a_{3}\right)$ of real-valued functions on $I$ a reduced connection.

Lemma 2.4 [BoMo]. There is a one-one correspondence between G-equivariant connections $\tilde{\omega}$ on $P_{X}$ and reduced connections. The correspondence is given by

$$
A_{\kappa}=\kappa^{*} s^{*} \tilde{\omega}=-a_{1} \beta^{1} \otimes l_{1}-a_{2} \beta^{2} \otimes l_{2}-a_{3} \beta^{3} \otimes l_{3},
$$

where $a$ is a reduced connection.

\footnotetext{
${ }^{6}$ Following the terminology of [Ur] and [BoMo], we use equivariant connection for what could as well be called an invariant connection
} 
Proof. This is an application of Urakawa's extension [Ur, Theorem 4.3] of a classical theorem of Wang on invariant connections.

The functions $a_{i}$ are related to the holonomy around certain loops on $X \subset S^{4}$. We consider $a_{3}$, but $a_{1}$ and $a_{2}$ are completely analogous. For fixed $\theta \in I$, we consider the path $\mu_{\theta}(t)=\left(\theta, \exp \left(t \varrho_{3} / 2\right)\right)$ on $Y$, where $t$ runs from 0 to $\pi$. The tangent vector $d / d t$ along the path is $l_{3}$. Under the projection (2.4) of $Y \rightarrow X$, the endpoints $\mu_{\theta}(0)$ and $\mu_{\theta}(\pi)$ project to $Q_{\theta} \in X$, so the path $\mu_{\theta}(t)$ projects to a closed loop on $X$.

We now calculate the holonomy around this loop. Lift the path $\mu_{\theta}(t)$ on $Y$ to a horizontal path $\left(\mu_{\theta}(t), h_{\theta}(t)\right)$ on $Y \times H=P_{Y}$. The differential equation for the lift,

$$
\frac{d h_{\theta}}{d t}-a_{3} l_{3} h_{\theta}=0 ; \quad h_{\theta}(0)=1
$$

is solved by

$$
h_{\theta}(t)=\exp \left(t a_{3} l_{3}\right)=\exp \left(t a_{3} \varrho_{3} / 2\right) \in H .
$$

However, we have $\left(\mu_{\theta}(\pi), h_{\theta}(\pi)\right) \sim\left(\mu_{\theta}(0), \varrho_{3} h_{\theta}(\pi)\right)$ under the equivalence $(2.11)$, so the holonomy around the closed loop on $X$ is

$$
\varrho_{3} h_{\theta}(\pi)=\exp \left(\left(a_{3}(\theta)+1\right) \pi \varrho_{3} / 2\right) \in H .
$$

For a given $\theta \in I$, this holonomy is trivial if and only if $a_{3}(\theta)=-1(\bmod 4)$.

The circumference of the closed path on $X$ is equal to $\pi f_{3}(\theta)=2 \pi \sin (\theta)$, which goes to zero as $\theta \rightarrow 0$. If the connection on $P_{X}$ over $X$ extends to a connection on a bundle $P$ over $S^{4}$, then the holonomy around a loop goes to the identity as the circumference of the loop goes to zero, which, by (2.11), requires

$$
\lim _{\theta \rightarrow 0} a_{3}(\theta)=-1(\bmod 4) .
$$

The same analysis as $\theta \rightarrow \pi / 3$ yields the condition

$$
\lim _{\theta \rightarrow \pi / 3} a_{2}(\theta)=-1(\bmod 4) .
$$

The special role of $a_{3}(0)$ and $a_{2}(\pi / 3)$ motivates the following:

Definition 2.5. For $(r, t) \in \mathbf{R}^{2}$, we say a reduced connection $a=\left(a_{1}, a_{2}, a_{3}\right)$ is a reduced $(r, t)$ connection if the limits

$$
a(0) \equiv \lim _{\theta \rightarrow 0} a(\theta), \quad a(\pi / 3) \equiv \lim _{\theta \rightarrow \pi / 3} a(\theta)
$$

exist, and furthermore $a(0)=(0,0, r)$ and $a(\pi / 3)=(0, t, 0)$.

Bor and Montgomery have established which equivariant connections on $P_{X}$ extend to the quadrupole bundle $P_{\left(n_{+}, n_{-}\right)}$. This is a special case of [Ur, Theorem 5.1].

Lemma 2.6 [BoMo, Proposition 3]. Let $(r, t)$ be two integers with $r=-1(\bmod 4)$ and $t=-1(\bmod 4)$, and with $n_{+}=|r| \neq 1$ and $n_{-}=|t| \neq 1$. Equation (2.20) gives a 1-1 correspondence between connections that extend to smooth equivariant connections on $P_{\left(n_{+}, n_{-}\right)}$and the reduced $(r, t)$ connections $\left(a_{1}, a_{2}, a_{3}\right)$ which have the following properties: Each $a_{i}$ can be extended to a smooth function on an open interval $(-\varepsilon, \pi / 3+\varepsilon), \varepsilon>0$, such that for all $x \in(-\varepsilon, \varepsilon)$,

$$
\begin{aligned}
a_{1}(x)=a_{2}(-x) ; & a_{3}(x)=a_{3}(-x) \\
a_{1}(\pi / 3+x)=a_{3}(\pi / 3-x) ; & a_{2}(\pi / 3+x)=a_{2}(\pi / 3-x) .
\end{aligned}
$$


Proof. The proof proceeds by analyzing the smoothness of the connection form in the local gauge induced from $\delta$. The details are given in [Ur, BoMo].

The case $n_{+}=1$, also worked out in [BoMo], is slightly more complicated. In that case, reduced connections satisfying the above conditions still give equivariant connections on the quadrupole bundle. However, there also exist equivariant connections for which the corresponding reduced connections may have nonzero values of $a_{1}(0)$ and $a_{2}(0)$. Similarly if $n_{-}=1$ then $a_{1}(\pi / 3)$ and $a_{3}(\pi / 3)$ may be nonzero. Such connections will not be used in this paper.

\subsection{The Yang-Mills Equations}

We now compute the Yang-Mills action and equations for an equivariant connection on $X$ in terms of the reduced connection $a$. We do this by lifting to the cover $Y$ of $X$, and working relative to the local section (gauge) $\kappa$. In the following, we often abbreviate $A_{\kappa}=A$. When discussing the $\operatorname{Lie}(H)$-valued connection and curvature forms, we will identify $\operatorname{Lie}(H)$ with the traceless hermitian $2 \times 2$ complex matrices, and the trace should be interpreted in this way.

The curvature 2-form on $Y$ is given by

$$
\begin{aligned}
F & =d A+A \wedge A \\
& =\left(-a_{1}^{\prime} d \theta \wedge \beta^{1}+\left(a_{1}+a_{2} a_{3}\right) \beta^{2} \wedge \beta^{3}\right) \otimes l_{1}+\text { (cyclic) },
\end{aligned}
$$

where' denotes $d / d \theta$. The Yang-Mills action of a smooth connection over $X$ is defined by

$$
S=\int_{X}-\operatorname{Tr}(* \tilde{F} \wedge \tilde{F})
$$

where $\tilde{F}$ is the curvature in any local gauge.

Proposition 2.7. Let $\tilde{\omega}$ be a smooth equivariant connection on $P_{X}$, and let $a=\left(a_{1}\right.$, $\left.a_{2}, a_{3}\right)$ be the corresponding reduced connection.

a) The action is given by

$$
S(\tilde{\omega})=S(a) \equiv \pi^{2} \int_{I}\left[\left(a_{1}^{\prime}\right)^{2} G_{1}+\left(a_{1}+a_{2} a_{3}\right)^{2} / G_{1}+(\text { cyclic })\right] d \theta .
$$

b) The self-duality equations $* F= \pm F$ read

$$
\begin{aligned}
& -a_{1}^{\prime} G_{1}= \pm\left(a_{1}+a_{2} a_{3}\right), \\
& -a_{2}^{\prime} G_{3}= \pm\left(a_{2}+a_{1} a_{3}\right), \\
& -a_{3}^{\prime} G_{3}= \pm\left(a_{3}+a_{1} a_{2}\right) .
\end{aligned}
$$

Proof. Using (2.7), we compute the Hodge dual of the curvature,

$$
* F=\left(-a_{1}^{\prime} G_{1} \beta^{2} \wedge \beta^{3}+\frac{\left(a_{1}+a_{2} a_{3}\right)}{G_{1}} d \theta \wedge \beta^{1}\right) \otimes l_{1}+(\text { cyclic }),
$$

which immediately gives (2.27) and

$$
\begin{aligned}
-\operatorname{Tr}(* F \wedge F)= & \frac{1}{2}\left[\left(a_{1}^{\prime}\right)^{2} G_{1}+\left(a_{1}+a_{2} a_{3}\right)^{2} / G_{1}+(\text { cyclic })\right] d \theta \\
& \wedge \beta^{1} \wedge \beta^{2} \wedge \beta^{3} .
\end{aligned}
$$


By gauge invariance, $\operatorname{Tr}(* F \wedge F)=s^{*} \operatorname{Tr}(* \tilde{F} \wedge \tilde{F})$. Now $s$ is an eightfold cover, so the integral over $Y$ is eight times the integral over $X$. Since $\int_{G} \beta^{3} \wedge \beta^{2} \wedge \beta^{2}=16 \pi^{2}$ (calculated by embedding $G \subset \mathbf{H}$ ), integrating (2.29) over $I \times G$ and dividing by 8 (for the 8-fold cover) gives (2.26).

The Yang-Mills equations

$$
0=D_{A} * F \equiv d * F+[A, * F]
$$

are the Euler-Lagrange equations of the action $S$. We call a smooth finite action reduced connection $a=\left(a_{1}, a_{2}, a_{3}\right)$ critical if

$$
\left.\frac{d}{d \varepsilon} S(a+\varepsilon c)\right|_{\varepsilon=0}=0
$$

for any $c=\left(c_{1}, c_{2}, c_{3}\right) \in C_{0}^{\infty}(I)$. If $a$ is a critical reduced connection, then integration by parts gives the Euler-Lagrange equations of $S$ expressed in terms of the reduced connection:

$$
\begin{aligned}
& \left(G_{1} a_{1}^{\prime}\right)^{\prime}=\left(a_{1}+a_{2} a_{3}\right) / G_{1}+a_{3}\left(a_{2}+a_{3} a_{1}\right) / G_{2}+a_{2}\left(a_{3}+a_{1} a_{2}\right) / G_{3}, \\
& \left(G_{2} a_{2}^{\prime}\right)^{\prime}=\left(a_{2}+a_{3} a_{1}\right) / G_{2}+a_{1}\left(a_{3}+a_{1} a_{2}\right) / G_{3}+a_{3}\left(a_{1}+a_{2} a_{3}\right) / G_{1}, \\
& \left(G_{3} a_{3}^{\prime}\right)^{\prime}=\left(a_{3}+a_{1} a_{2}\right) / G_{3}+a_{2}\left(a_{1}+a_{2} a_{3}\right) / G_{1}+a_{1}\left(a_{2}+a_{3} a_{1}\right) / G_{2} .
\end{aligned}
$$

We call these equations the reduced Yang-Mills equations. This terminology is justified by

Proposition 2.8. A smooth finite-action equivariant connection on $P_{X} \rightarrow X$ is a solution of the Yang-Mills equations on $X$ iff the reduced connection satisfies the reduced Yang-Mills equations.

Proof. This can be proved with the principle of symmetric criticality [Pal]. However, we prove this explicitly for $A=A_{\kappa}$. For a smooth YM connection on $X$, we use (2.28), and take the exterior derivative of $* F$ using the Maurer-Cartan equations, obtaining

$$
\begin{aligned}
D_{A} * F= & {\left[-\left(G_{1} a_{1}^{\prime}\right)^{\prime}+\frac{a_{1}+a_{2} a_{3}}{G_{1}}+\frac{a_{3}\left(a_{2}+a_{1} a_{3}\right)}{G_{2}}+\frac{a_{2}\left(a_{3}+a_{1} a_{2}\right)}{G_{3}}\right.} \\
& \times d \theta \wedge \beta^{2} \wedge \beta^{3} \otimes l_{1}+(\text { cyclic }) .
\end{aligned}
$$

The vanishing of $D_{A} * F$ on $X$ is thus equivalent to (2.31).

We define $C_{2}$ to be the integral over $X \subset S^{4}$ of the second Chern form,

$$
C_{2} \equiv \int_{X} \frac{-\operatorname{Tr}(\tilde{F} \wedge \tilde{F})}{8 \pi^{2}}
$$

Since $X$ is an open manifold, $C_{2}$ need not be an integer, depending on the holonormy around the two-dimensional singular sets [FHP1, FHP2].

Proposition 2.9. If $a=\left(a_{1}, a_{2}, a_{3}\right)$ is a finite action reduced $(r, t)$ connection, then $C_{2}=\left(r^{2}-t^{2}\right) / 8$. 
Proof.

$$
\begin{aligned}
& \operatorname{Tr}(F \wedge F)=\left(a_{1}^{\prime}\left(a_{1}+a_{2} a_{3}\right)+\text { cyclic }\right) d \theta \wedge \beta^{1} \wedge \beta^{2} \wedge \beta^{3} \\
& \quad=\frac{1}{2} d\left(a_{1}^{2}+a_{2}^{2}+a_{3}^{2}+2 a_{1} a_{2} a_{3}\right) \wedge \beta^{1} \wedge \beta^{2} \wedge \beta^{3} . \\
& C_{2} \equiv-\frac{1}{8 \pi^{2}} \int_{S^{4}} \operatorname{Tr}(\tilde{F} \wedge \tilde{F}) \\
& =-\frac{1}{64 \pi^{2}} \int_{I \times G} \operatorname{Tr}(F \wedge F) \\
& =-\frac{1}{64 \pi^{2}} \int_{I} \frac{1}{2} d\left(a_{1}^{2}+a_{2}^{2}+a_{3}^{2}+2 a_{1} a_{2} a_{3}\right) \int_{G} \beta^{2} \wedge \beta^{2} \wedge \beta^{3} \\
& =-\left[\left(a_{2}(\pi / 3)\right)^{2}-\left(a_{3}(0)\right)^{2}\right] / 8=\left(r^{2}-t^{2}\right) / 8 .
\end{aligned}
$$

If $r=-1(\bmod 4)$ and $t=-1(\bmod 4)$ then the holonomy vanishes and $C_{2}$ is an integer. Together with Lemma 2.6 this yields the formula $C_{2}=\left(n_{+}^{2}-n_{-}^{2}\right) / 8$ for the Chern number of the quadrupole bundle $P_{\left(n_{+}, n_{-}\right)} \rightarrow S^{4}$.

\section{Existence of Yang-Mills Connections}

In this chapter we prove an existence theorem for reduced $(r, t)$ connections:

Theorem 3.1. For any $(r, t) \in \mathbf{R}^{2}$ with $|r|>1,|t|>1$, there exists a smooth reduced $(r, t)$ connection that is a finite-action solution of the reduced Yang-Mills equations on $I$, and which is not (anti)self-dual.

There is a discrete symmetry of the reduced action (2.26), the (anti)self-dual equations (2.27) and the reduced Yang-Mills equations (2.31). These are all invariant under the change of sign of any two of the functions $a_{i}$. That is, the four distinct reduced connections

$$
\left(a_{1}, a_{2}, a_{3}\right), \quad\left(-a_{1},-a_{2}, a_{3}\right), \quad\left(-a_{1}, a_{2},-a_{3}\right), \quad\left(a_{1},-a_{2},-a_{3}\right)
$$

all have the same action, and if any one is a solution of the reduced self-dual or Yang-Mills equations, then all four are solutions. It is therefore sufficient to prove Theorem 3.1 for $r$ and $t$ positive.

\subsection{The Hilbert Space of Reduced Connections}

We first define a class of weighted Sobolev spaces on $I$. Let $\mathscr{A}=C(\bar{I}) \cap C^{\infty}(I)$, and let $F$ be a continuous positive function on $I$. For $b \in \mathscr{A}$, define the norm

$$
\|b\|_{F} \equiv\left(\int_{0}^{\pi / 3} d x\left[\left(b^{\prime}\right)^{2} F+(b)^{2} / F\right]\right)^{1 / 2}
$$

and consider the subspace $\mathscr{C}_{F}$ of functions with finite norm. We define the real Hilbert space $\mathscr{H}_{F}$ as the completion of $\mathscr{C}_{F}$ with respect to the norm $\|\cdot\|_{F}$. 
We next consider affine subspaces. Define

$$
C(\bar{I} ; \alpha, \beta)=\{b \in C(\bar{I}) ; b(0)=\alpha, b(\pi / 3)=\beta\},
$$

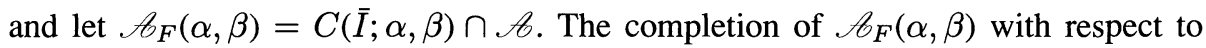
$\|\cdot\|_{F}$ is a closed affine subspace of $\mathscr{H}_{F}$, which we denote by $\mathscr{H}_{F}(\alpha, \beta)$. Note that $\mathscr{H}_{F}(0,0)$ is a vector subspace of $\mathscr{H}_{F}$.

We now prove a Sobolev-type lemma.

Lemma 3.2. $\mathscr{H}_{F}(\alpha, \beta)$ embeds continuously into $C(\bar{I} ; \alpha, \beta)$.

Proof. Given $a, c \in \mathscr{A}_{F}(\alpha, \beta)$, we have $b=(a-c) \in \mathscr{C}_{F}(0,0)$,

$$
\begin{aligned}
{[b(x)]^{2} } & =2 \int_{0}^{x} b^{\prime}(y) b(y) d y \\
& =2 \int_{0}^{x}\left(b^{\prime} \sqrt{F}\right)(b / \sqrt{F}) d y \\
& \leq 2\left(\int_{0}^{x}\left(b^{\prime}\right)^{2} F d y\right)^{1 / 2}\left(\int_{0}^{x} \frac{b^{2} d y}{F}\right)^{1 / 2} \\
& \leq 2\|b\|_{F}^{2} .
\end{aligned}
$$

Taking the supremum over $x \in \bar{I}$,

$$
\|b\|_{C(\bar{I})} \leq \sqrt{2}\|b\|_{F},
$$

so $\mathscr{C}_{F}(\alpha, \beta)$ embeds continuously into $C(\bar{I})$, in fact into the closed subspace $C(\bar{I} ; \alpha, \beta)$. Now $\mathscr{A}_{F}(\alpha, \beta)$ is dense in $\mathscr{H}_{F}(\alpha, \beta)$, so we are done.

Note that under the reparametrization $T=\int_{\pi / 6}^{x} d y / F(y)$, the $\mathscr{H}_{F}$ norm becomes

$$
\|b\|_{F}=\int_{-\gamma}^{\delta}\left[(d b / d T)^{2}+b^{2}\right] d T
$$

which is the Sobolev $W_{1}^{2}$ norm on the interval $(\delta, \gamma)$ where $\gamma=\int_{0}^{\pi / 6} d x / F(x)$ and $\delta=\int_{\pi / 6}^{\pi / 3} d x / F(x)$. If $b \in \mathscr{H}_{F}$ and $\gamma$ is infinite, then $\lim _{x \rightarrow 0} b(x)=0$, and if $\delta$ is infinite then $\lim _{x \rightarrow \pi / 3} b(x)=0$.

Definition 3.3. The Hilbert space $\mathscr{H}$ is the orthogonal sum

$$
\mathscr{H} \equiv \mathscr{H}_{G_{1}} \oplus \mathscr{H}_{G_{2}} \oplus \mathscr{H}_{G_{3}} .
$$

The affine subspace $\mathscr{H}(r, t)$ of $\mathscr{H}$ is the sum of affine subspaces

$$
\mathscr{H}(r, t) \equiv \mathscr{H}_{G_{1}}(0,0) \oplus \mathscr{H}_{G_{2}}(0, t) \oplus \mathscr{H}_{G_{3}}(r, 0) .
$$


By the discussion preceding Definition 3.3, all elements $a \in \mathscr{H}$ have $a_{1}(0)=a_{2}(0)=$ 0 and $a_{1}(\pi / 3)=a_{3}(\pi / 3)=0$, so $\mathscr{H}=\bigcup_{r, t} \mathscr{H}(r, t)$. We shall show that if $|r| \neq 1$ and $|t| \neq 1$, then all finite-action reduced $(r, t)$ connections belong to $\mathscr{H}(r, t) \subset \mathscr{H}$.

For a smooth $a \in \mathscr{H}$, we define the action $S(a)$ as in (2.26), and divide it into two pieces

$$
S(a)=\pi^{2}\left(S_{1}(a)+S_{2}(a)\right)=\pi^{2} \int_{0}^{\pi / 3}\left[s_{1}(a)+s_{2}(a)\right] d x
$$

where

$$
\begin{aligned}
& s_{1}(a) \equiv\left[\left(a_{1}^{\prime}\right)^{2} G_{1}+\left(a_{2}^{\prime}\right)^{2} G_{2}+\left(a_{3}^{\prime}\right)^{2} G_{3}\right] \\
& s_{2}(a) \equiv\left[\left(a_{1}+a_{2} a_{3}\right)^{2} / G_{1}+\left(a_{2}+a_{1} a_{3}\right)^{2} / G_{2}+\left(a_{3}+a_{1} a_{2}\right)^{2} / G_{3}\right] .
\end{aligned}
$$

Now the smooth reduced connections are dense in $\mathscr{H}$, and there is a unique normcontinuous extension of $S_{1}$ to $\mathscr{H}$, since $S_{1}(a) \leq \pi^{2}\|a\|_{\mathscr{H}}^{2}$. By Lemma 3.2, any $a \in \mathscr{H}$ is continuous, so $S_{2}$ is well-defined (though possibly infinite) on $\mathscr{H}$. It is clear that $S_{1}$ and $S_{2}$ are nonnegative.

A minimizing sequence in $\mathscr{H}(r, t)$ is a sequence of reduced connections $A^{(n)} \in$ $\mathscr{H}(r, t)$ such that

$$
\lim _{n \rightarrow \infty} S\left(a^{(n)}\right)=\inf _{b \in \mathscr{H}(r, t)} S(b) .
$$

A minimizing sequence obviously exists for any $(r, t)$.

\subsection{The Limiting Connection}

For simplicity, in the remainder of this chapter we only consider positive $r$ and $t$. By the discrete symmetry (3.1), these results are easily extended to $r$ and $t$ of arbitrary sign. In this section we establish

Proposition 3.4. For positive $r \neq 1$ and positive $t \neq 1$, every minimizing sequence in $\mathscr{H}(r, t)$ has a weakly convergent subsequence. The weak limit $a^{*}$ of this subsequence belongs to $\mathscr{H}(r, t)$.

We first prove two technical lemmas.

Lemma 3.5. Let $a$ be a smooth reduced $(r, t)$ connection with finite action $S(a)=$ $\pi^{2} M$. Then there exists a function $K_{3}(x)$ such that

$$
\left|a_{3}(x)-r\right| \leq\left(K_{3}(x) \int_{0}^{x} \frac{d y}{G_{3}(y)}\right)^{1 / 2}
$$

with $0 \leq K_{3}(x) \leq M$ and $\lim _{x \rightarrow 0} K_{3}(x)=0$. Similarly, there exists a function $K_{2}(x)$ such that

$$
\left|a_{2}(x)-r\right| \leq\left(K_{2}(x) \int_{x}^{\pi / 3} \frac{d y}{G_{2}(y)}\right)^{1 / 2}
$$

where $0 \leq K_{2}(x) \leq M$, and $\lim _{x \rightarrow \pi / 3} K_{2}(x)=0$. 
Proof.

$$
\begin{aligned}
\left|a_{3}(x)-a_{3}(0)\right|^{2} & =\left|\int_{0}^{x} a_{3}^{\prime}(y) d y\right|^{2} \\
& \leq\left(\int_{0}^{x}\left(a_{3}^{\prime} \sqrt{G_{3}}\right)^{2} d y\right)\left(\int_{0}^{x}\left(1 /{\sqrt{G_{3}}}^{2} d y\right)\right. \\
& =K_{3}(x) \int_{0}^{x} \frac{d y}{G_{3}(y)},
\end{aligned}
$$

where we have used the Schwartz inequality. The proof of (3.12) is similar.

Using Lemma 3.2 and the fact that smooth reduced connections are dense in $\mathscr{H}(r, t)$, we have

Corollary 3.6. If $a \in \mathscr{H}(r, t)$ has finite action $S(a)=\pi^{2} M$, then (3.11) and (3.12) hold.

Lemma 3.7. For positive $r \neq 1$ and positive $t \neq 1$, and any finite $M$, the set

$$
\mathscr{B}(r, t, M) \equiv\left\{a \in \mathscr{H}(r, t) ; S(a) \leq \pi^{2} M\right\}
$$

is a norm-bounded subset of $\mathscr{H}$.

Proof. For a connection $a=\left(a_{1}, a_{2}, a_{3}\right) \in \mathscr{H}(r, t)$ with action $S(a) \leq \pi^{2} M$, we wish to bound the squared norm

$$
\begin{aligned}
\|a\|_{\mathscr{H}}^{2} & =\int_{0}^{\pi / 3}\left[\left(a_{1}^{\prime}\right)^{2} G_{1}+\left(a_{2}^{\prime}\right) G_{2}+\left(a_{3}^{\prime}\right)^{2} G_{3}+\frac{a_{1}^{2}}{G_{1}}+\frac{a_{2}^{2}}{G_{2}}+\frac{a_{3}^{2}}{G_{3}}\right] d x \\
& =S_{1}(a)+H(a),
\end{aligned}
$$

where

$$
H(a) \equiv \int_{0}^{\pi / 3} h(a) d x=\int_{0}^{\pi / 3}\left[\frac{a_{1}^{2}}{G_{1}}+\frac{a_{2}^{2}}{G_{2}}+\frac{a_{3}^{3}}{G_{3}}\right] d x .
$$

The bound should depend only on $M, r$, and $t . S_{1}, S_{2}$ and $H$ are all nonnegative on $\mathscr{H}$, so we immediately obtain $S_{1}(a) \leq M$.

Bounding $H(a)$ is more complicated due to the presence of cubic and quartic terms. We divide $I=(0, \pi / 3)$ into three subintervals, and bound $H$ separately on each interval. Take

$$
\alpha=\frac{1}{2} \min \left(M^{-1 / 2}|r-1|, M^{-1 / 2}|t-1|, \pi / 6\right), \quad \beta=\pi / 3-\alpha,
$$

and let $I_{1}=(0, \alpha), I_{2}=[\alpha, \beta]$, and $I_{3}=(\beta, \pi / 3)$.

We begin with $I_{1}$, where

$$
\frac{1}{4 x} \leq \frac{1}{G_{1,2}(x)} \leq \frac{1}{x} ; \quad x \leq \frac{1}{G_{3}(x)} \leq 2 x .
$$


By Eq. (3.11), this gives

$$
\left|a_{3}(x)-r\right| \leq M^{1 / 2} x \leq \frac{|r-1|}{2},
$$

where we have used $x \leq \alpha \leq M^{-1 / 2}|r-1| / 2$. Thus

$$
\left|a_{3}\right| \leq r+\frac{|r-1|}{2} \leq \frac{3 r+2}{2} .
$$

Combining (3.15) and (3.17) yields the bound

$$
\int_{0}^{\alpha}\left[\left(a_{3}\right)^{2} / G_{3}\right] d x \leq \alpha^{2}(3 r+2)^{2} / 4 .
$$

We now bound the remaining two terms on $I_{1}$. From (3.16) and the triangle inequality

$$
\left|a_{3} \pm 1\right| \geq|r-1| / 2 \text {. }
$$

Using this fact, and using (3.15) repeatedly, we have

$$
\begin{aligned}
\int_{0}^{\alpha} & {\left[\left(a_{1}\right)^{2} / G_{1}+\left(a_{2}\right)^{2} / G_{2}\right] d x } \\
& \leq \int_{0}^{\alpha}\left[\left(a_{1}\right)^{2}+\left(a_{2}\right)^{2}\right] \frac{d x}{x} \\
& =\frac{1}{2} \int_{0}^{\alpha}\left[\left(a_{1}+a_{2}\right)^{2}+\left(a_{1}-a_{2}\right)^{2}\right] \frac{d x}{x} \\
& \leq \frac{2}{(r-1)^{2}} \int_{0}^{\alpha}\left[\left(a_{3}+1\right)^{2}\left(a_{1}+a_{2}\right)^{2}+\left(a_{3}-1\right)^{2}\left(a_{1}-a_{2}\right)^{2}\right] \frac{d x}{x} \\
& =\frac{4}{(r-1)^{2}} \int_{0}^{\alpha}\left[\left(a_{1}+a_{2} a_{3}\right)^{2}+\left(a_{2}+a_{1} a_{3}\right)^{2}\right] \frac{d x}{x} \\
& \leq \frac{16}{(r-1)^{2}} \int_{0}^{\alpha}\left[\left(a_{1}+a_{2} a_{3}\right)^{2} / G_{1}+\left(a_{2}+a_{1} a_{3}\right)^{2} / G_{2}\right] d x \\
& \leq \frac{16 M}{(r-1)^{2}} \cdot
\end{aligned}
$$

For $r \neq 1$, this is finite, and adding (3.18) and (3.19), we obtain a bound on $\int_{I_{1}} h(a) d x$. Analogous techniques bound $\int_{I_{3}} h(a) d x$.

Finally, we bound $\int_{I_{2}} h(a) d x$. For any $x \leq \beta$, we have $1 / G_{3}(x) \leq 1 / \alpha$, and we obtain from Eq. (3.11)

$$
\left|a_{3}(x)-r\right| \leq\left(\frac{M \pi}{3 \alpha}\right)^{1 / 2}
$$


and, by the triangle inequality,

$$
\left|a_{3}\right| \leq K_{+} \equiv\left(\frac{M \pi}{3 \alpha}\right)^{1 / 2}+r
$$

yielding

$$
\int_{\alpha}^{\beta}\left[\left(a_{3}\right)^{2} / G_{3}\right] d x \leq \frac{\pi}{3 \alpha} K_{+}^{2}
$$

Similarly, we obtain on $I_{2}$ the uniform bound

$$
\left|a_{2}\right| \leq K_{-} \equiv\left(\frac{M \pi}{3 \alpha}\right)^{1 / 2}+t
$$

and the analogous bound on the $\left(a_{2}\right)^{2}$ piece of the norm. Only the $\left(a_{1}\right)^{2}$ term on $I_{2}$ remains. The triangle inequality gives

$$
\left|a_{1}\right| \leq\left|a_{1}+a_{2} a_{3}\right|+K^{2}
$$

where $K \equiv \max \left(K_{+}, K_{-}\right)$, and squaring gives

$$
\left|a_{1}\right|^{2} \leq\left|a_{1}+a_{2} a_{3}\right|^{2}+2 K^{2}\left|a_{1}+a_{2} a_{3}\right|+K^{4} .
$$

Integrating this inequalities gives

$$
\begin{aligned}
\int_{\alpha}^{\beta} \frac{\left(a_{1}\right)^{2}}{G_{1}} d x & \leq \int_{\alpha}^{\beta} \frac{\left|a_{1}+a_{2} a_{3}\right|^{2}}{G_{1}} d x+2 K^{2} \int_{\alpha}^{\beta} \frac{\left|a_{1}+a_{2} a_{3}\right|}{G_{1}} d x+K^{4} \int_{\alpha}^{\beta} \frac{d x}{G_{1}(x)} \\
& \leq M+2 K^{2}\left(M \int_{\alpha}^{\beta} \frac{d x}{G_{1}(x)}\right)^{1 / 2}+K^{4} \int_{\alpha}^{\beta} \frac{d x}{G_{1}(x)}
\end{aligned}
$$

where we have used the Schwartz inequality

$$
\left|\int_{\alpha}^{\beta} \frac{\left|a_{1}+a_{2} a_{3}\right|}{G_{1}} d x\right|^{2} \leq\left(\int_{\alpha}^{\beta} \frac{\left|a_{1}+a_{2} a_{3}\right|^{2}}{G_{1}} d x\right)\left(\int_{\alpha}^{\beta} \frac{1}{G_{1}} d x\right) .
$$

This completes the bounding of $\int_{I_{2}} h(a) d x$.

Adding up the previous bounds, we obtain a bound on $\|a\|_{\mathscr{H}}^{2}=S_{1}(a)+H(a)$ that depends only on $M, r$, and $t$

Proof of Proposition 3.4. Any minimizing sequence $a^{(n)}$ in $\mathscr{H}(r, t)$ is by definition a set of bounded action. By Lemma 3.7, such a set lies within a sufficiently large finite ball in $\mathscr{H}$. By the Banach-Alaoglu theorem and the reflexivity of Hilbert space, a finite ball is weakly compact [RS, Theorem IV.21], so the minimizing sequence has a weakly convergent subsequence in $\mathscr{H}$. 
Now $\mathscr{H}(r, t)$ is a norm-closed convex subset of $\mathscr{H}$, so it is weakly closed ([RS], problem 12 , p. 388). The weak limit $a^{*}$ of the convergent subsequence in $\mathscr{H}(r, t)$ thus belongs to $\mathscr{H}(r, t)$.

\subsection{Weak Lower Semicontinuity}

We next show that the weak limiting connection $a^{*}$ on $\mathscr{H}(r, t)$ attains the infimum of the action on $\mathscr{H}(r, t)$, by showing that the action $S$ is sequentially weakly lower semicontinuous on $\mathscr{H}$. That is, if $b^{(n)} \neg b^{*}$ in $\mathscr{H}$ is a weakly convergent sequence, then

$$
S\left(b^{*}\right) \leq \underline{\lim } S\left(b^{(n)}\right) .
$$

For $0<\delta<\pi / 6$ we denote by $I_{\delta}$ the open interval $(\delta, \pi / 3-\delta) \subset I$. Let $W_{1}^{2}\left(I_{\delta}\right)$ be the ordinary (unweighted) Sobolev space with norm

$$
\|g\|_{W_{1}^{2}\left(I_{\delta}\right)}^{2}=\int_{\delta}^{\pi / 3-\delta}\left[\left(g^{\prime}\right)^{2}+g^{2}\right] d x .
$$

Lemma 3.8. Let $b^{(n)} \rightarrow b^{*}$ be a weakly convergent sequence in $\mathscr{H}$. Then the functions $b_{i}^{(n)}, i=1,2,3$ converge to $b_{i}^{*}$ uniformly on $\bar{I}_{\delta} \subset I$.

Proof. Since the functions $G_{i}$ and $1 / G_{i}$ are bounded on $I_{\delta}$, the restriction map

$$
\begin{aligned}
T: \mathscr{H}_{G_{i}} & \rightarrow W_{1}^{2}\left(I_{\delta}\right) \\
b_{i} & \left.\mapsto b_{i}\right|_{I_{\delta}},
\end{aligned}
$$

is bounded, and thus continuous.

The map $T$ then takes a weakly convergent sequence $b_{i}^{(n)} \rightarrow b_{i}^{*}$ in $\mathscr{H}_{G_{i}}$, to a weakly convergent sequence in $W_{1}^{2}\left(I_{\delta}\right)$. We now use the Kondrakov-Rellich embedding theorem [Au, Theorem 2.33; Ad, Chap. VI] to conclude that $W_{1}^{2}\left(I_{\delta}\right)$ embeds compactly into $C\left(\bar{I}_{\delta}\right)$. A compact map takes a weakly convergent sequence to a norm convergent sequence [RS, Theorem VI.11], so $b_{i}^{(n)}$ converges to $b_{i}^{*}$ in the uniform norm on $I_{\delta}$.

Proposition 3.9. The action $S$ is weakly lower semicontinuous on $\mathscr{H}$.

Proof. Recall the splitting (3.8) of $S=\pi^{2}\left(S_{1}+S_{2}\right) . S_{1}$ is convex, and thus weakly lower semicontinuous ([RS], p.355).

It remains to show that $S_{2}$ is weakly lower semicontinuous. Suppose that $S_{2}$ were not. Then there would exist a weakly convergent sequence $b^{(n)} \rightarrow b^{*}$ in $\mathscr{H}$ such that $S_{2}\left(b^{*}\right)>\underline{\lim } S_{2}\left(b^{(n)}\right)$. For such a sequence,

$$
\varepsilon \equiv S_{2}\left(b^{*}\right)-\underline{\lim } S_{2}\left(b^{(n)}\right)>0 .
$$

Since

$$
S_{2}\left(b^{*}\right)=\lim _{\delta \rightarrow 0} \int_{\delta}^{\pi / 3-\delta} s_{2}\left(b^{*}\right) d x
$$


there exists a $\delta>0$ such that

$$
\begin{aligned}
\varepsilon / 2 & \leq \int_{\delta}^{\pi / 3-\delta} s_{2}\left(b^{*}\right) d x-\underline{\lim } \int_{0}^{\pi / 3} s_{2}\left(b^{(n)}\right) d x \\
& \leq \underline{\lim } \int_{\delta}^{\pi / 3-\delta}\left[s_{2}\left(b^{*}\right)-s_{2}\left(b^{(n)}\right)\right] d x \\
& =0 .
\end{aligned}
$$

Here we have used the fact that $s_{2}\left(b^{(n)}\right)$ is nonnegative, and Lemma 3.8. We have a contradiction, which proves that $S_{2}$ is weakly lower semicontinuous.

We now obtain the existence of minimizers.

Theorem 3.10. For positive $r \neq 1$ and positive $t \neq 1$, there exists a reduced $(r, t)$ connection that is a smooth finite-action solution of the reduced Yang-Mills equations.

Proof. Let $a^{*}$ be the weak limiting reduced connection of Proposition 3.4. Then by Proposition $3.9, a^{*}$ minimizes the action on $\mathscr{H}(r, t)$. So $a^{*}$ is a weak solution of the Euler-Lagrange equations on $I$. This is a semilinear elliptic system of ODE's, and standard regularity theory (see e.g. [Ber], Sect. 1.5) gives the smoothness of the $a_{i}$ on $I$.

\subsection{Nonexistence of (Anti)Self-Dual Solutions}

Finally, we show that these solutions are not (anti)self-dual.

Theorem 3.11. There are no finite-action anti-self-dual reduced $(r, t)$ connections with $r>1$. There are no finite-action self-dual reduced $(r, t)$ connections with $t>1$.

Proof. We will prove the first statement; the proof of the second statement is similar.

A reduced $(r, t)$ connection has $a_{1}(0)=a_{2}(0)=a_{1}(\pi / 3)=a_{3}(\pi / 3)=0$. We will show that a solution to the anti-self-dual equations must have $a_{3}(\pi / 3)>0$, contradicting this.

The anti-self-duality equations are

$$
\begin{aligned}
& a_{1}^{\prime}=\left(a_{1}+a_{2} a_{3}\right) / G_{1}, \\
& a_{2}^{\prime}=\left(a_{2}+a_{1} a_{3}\right) / G_{2}, \\
& a_{3}^{\prime}=\left(a_{3}+a_{1} a_{2}\right) / G_{3} .
\end{aligned}
$$

If at some point $a_{3}$ is positive and $a_{1}$ and $a_{2}$ are nonnegative, then all three derivatives are nonnegative, so $a_{3}$ stays positive and $a_{1,2}$ remain nonnegative as $\theta$ increases. Similarly, if $a_{1}, a_{2} \leq 0<a_{3}$, then $a_{1}^{\prime}, a_{2}^{\prime} \leq 0<a_{3}^{\prime}$, and again the signs persist. In either case $a_{3}(\pi / 3)$ will not be zero. Thus it suffices to find a $\theta \in I$, where $a_{1}$ and $a_{2}$ do not have opposite signs, and where $a_{3}>0$.

Since $a_{3}(0)=r>1$ and $a_{3}$ is continuous, $a_{3}>1$ on some nonempty interval $(0, \varepsilon)$. Suppose $a_{1}$ and $a_{2}$ had opposite signs throughout this interval. Then $\left|a_{1}-a_{2}\right|>$ 
$\left|a_{1}+a_{2}\right|$ on this interval. The Eq. (3.32) yield

$$
\begin{aligned}
\frac{d\left(a_{1}-a_{2}\right)^{2}}{d \theta}= & 2\left(a_{1}-a_{2}\right)\left(a_{1}^{\prime}-a_{2}^{\prime}\right) \\
= & -\left(\frac{1}{G_{1}}+\frac{1}{G_{2}}\right)\left(a_{3}-1\right)\left(a_{1}-a_{2}\right)^{2} \\
& +\left(\frac{1}{G_{1}}-\frac{1}{G_{2}}\right)\left(a_{3}+1\right)\left(a_{1}-a_{2}\right)\left(a_{1}+a_{2}\right) .
\end{aligned}
$$

Since $\left(G_{1}^{-1}+G_{2}^{-1}\right)=O\left(\theta^{-1}\right)$ and $G_{1}^{-1}-G_{2}^{-1}=O(1)$, since $a_{3}-1$ is bounded away from zero on $(0, \varepsilon)$, and since $\left|a_{1}-a_{2}\right|>\left|a_{1}+a_{2}\right|$, the first term is larger than the second for $\theta$ sufficiently small. Thus $d\left(a_{1}-a_{2}\right)^{2} / d \theta$ is negative on some nonempty interval $(0, \delta) \subseteq(0, \varepsilon)$, so $\left(a_{1}-a_{2}\right)^{2}$ is a positive decreasing function. However, $a_{1}(0)=a_{2}(0)=0$, which is a contradiction. Thus $a_{1}$ and $a_{2}$ cannot be of opposite signs on all of $(0, \delta)$.

Proof of Theorem 3.1. For $r>1$ and $t>1$, Theorem 3.10 yields the existence of a reduced $(r, t)$ connection that is a solution of the reduced YM equations, and Theorem 3.11 shows that this solution cannot be (anti)self-dual. The discrete symmetry (3.1) completes the proof.

\section{Regularity on the Four-Sphere}

We have established the existence of smooth equivariant non-self-dual Yang-Mills connections on the bundle $P_{X} \rightarrow X$, corresponding to reduced $(r, t)$ connections for all values of $(r, t)$ with $|r|>1,|t|>1$. In this chapter, we complete the proof of Theorem 1.1 by showing that, for the appropriate values of $(r, t)$, these connections extend to smooth YM connections on quadrupole bundles $P_{\left(n_{+}, n_{-}\right)} \rightarrow S^{4}$.

Theorem 4.1. Let $r=-1(\bmod 4)$ and $t=-1(\bmod 4)$, with $n_{+} \equiv|r| \neq 1$ and $n_{-} \equiv|t| \neq 1$. A finite-action reduced $(r, t)$ connection that satisfies the reduced YangMills equations corresponds to an equivariant connection on $P_{X} \rightarrow X$ that extends to a smooth Yang-Mills connection on the quadrupole bundle $P_{\left(n_{+}, n_{-}\right)} \rightarrow S^{4}$.

The proof of Theorem 4.1 is an application of the following theorem of Uhlenbeck [Uh2], which adapted to our situation states:

Theorem 4.2. Let $\tilde{A}$ be a weak solution of the Yang-Mills equations on a neighbourhood $U$ of $Q_{0}$ in $S^{4}$, and suppose that $\tilde{A} \in W_{1}^{2}(U)$. Then on some neighborhood $\tilde{U}$ of $Q_{0}$ in $S^{4}, \tilde{A}$ is equivalent by a gauge transformation $\phi \in W_{2}^{2}(\tilde{U})$ to a smooth oneform $A$.

Proof. See [Uh2], Theorem 1.3 and Corollary 1.4]. The theorem proved by demonstrating the existence of a gauge transformation $\phi$ for which the transformed connection form $A$ is in Coulomb gauge, $d^{*} A=0$. The Yang-Mills equations together with the Coulomb gauge condition are a uniformly elliptic system of PDE's, and elliptic regularity theory applies. Alternatively, we could use Theorem 5.1 and Corollary 5.2 of [SiSi2], which are specifically suited to a codimension two singular set.

The proof of Theorem 4.1 consists of two parts. First, we will show that finiteaction reduced connections extend from equivariant connections on $P_{X} \rightarrow X$ to continuous connections on the appropriate quadrupole bundle $P_{\left(n_{+}, n_{-}\right)} \rightarrow S^{4}$. Then 
we will show that if the reduced connection is a solution of the reduced Yang-Mills equations, then the conditions of Theorem 4.2 are satisfied in the local gauge induced by $\delta$ on $S^{4}$. Since $\tilde{A}$ is continuous and $A$ is smooth the gauge transformation $\phi$, which could a priori be a noncontinuous element of $W_{2}^{2}$, must be continuously differentiable.

Unfortunately, the technical details of the regularity proof are somewhat lengthy. In the next section, we present a simple regularity result which illustrates the ideas used in the proof of Theorem 4.1, while avoiding the complications of gauge invariance and higher dimensions. The following section is not essential for the proof of Theorem 4.1.

\subsection{A Simple Regularity Result}

We consider as an illustrative example the probolem of extending harmonic functions from the punctured two-disc to the unpunuctured two-disc. Let $\mathrm{B}$ be the open unit disc in $\mathbf{R}^{2}$ with Cartesian coordinates $(x, y)$, and let $B_{0}$ be the punctured unit disc $B-\{0\}$. Let $E$ be the energy functional

$$
E(f) \equiv \int_{B_{0}}|d f|^{2} d x \wedge d y .
$$

The critical points of $E$ are the harmonic functions, which satisfy $\Delta f=0$. The following is a simple analog of Theorem 4.1:

Proposition 4.3. Let $f$ be a bounded finite-energy harmonic function on $B_{0}$. Then $f$ extends to a smooth function on $B$.

Note that this example has a singular set of codimension two, the same codimension as the singular (two-dimensional) $G$-orbits in $S^{4}$. Codimension 2 plays a crucial role, and Proposition 4.3 is false on the one-dimensional ball, the function $f(x)=x /|x|$ being a counterexample.

Proof of Proposition 4.3. A standard regularity result [LU, Chap. 3; Ber, Sect. 1.5] states that if $f \in W_{1}^{2}(B)$ is bounded and weakly harmonic on $B$, then $f \in C^{\infty}(B)$. Given a bounded harmonic function $f$ on $B_{0}$ which has finite energy, we first prove that $f \in W_{1}^{2}(B)$, and then that $f$ is weakly harmonic on $B$. Proposition 4.3 is then immediate.

It follows from the boundedness and finite energy of $f$ that $f \in W_{1}^{2}\left(B_{0}\right)$. To show that $f \in W_{1}^{2}(N)$ we need to show that the function $\partial_{x} f$, defined a priori only on $B_{0}$, is the weak derivative of $f$ on $B$, i.e., that

$$
\int_{B}\left(\partial_{x} \phi\right) f d x \wedge d y=-\int_{B} \phi\left(\partial_{x} f\right) d x \wedge d y
$$

for any $\phi \in C_{0}^{\infty}(B)$. For fixed $y \neq 0$, integration by parts gives

$$
\int\left(\partial_{x} \phi\right) f d x=-\int \phi\left(\partial_{x} f\right) d x .
$$

Integrating this expression over $y$, using Fubini's theorem and the fact that the line $y=0$ has zero measure, we see that Eq. (4.2) holds, so $f \in W_{1}^{2}(B)$.

We now show that $f$ is weakly harmonic on $B$, i.e.,

$$
0=\int_{B}\left(\left(\partial_{x} f\right)\left(\partial_{x} \phi\right)+\left(\partial_{y} f\right)\left(\partial_{y} \phi\right)\right) d x \wedge d y
$$


for any $\phi \in C_{0}^{\infty}(B)$. For fixed $y \neq 1$, integrating by parts,

$$
\int\left(\partial_{x} f\right)\left(\partial_{x} \phi\right) d x=-\int\left(\partial_{x} \partial_{x} f\right) \phi d x .
$$

As before, we integrate this expression over $y$, using Fubini's theorem and the fact that the line $y=0$ has zero measure, obtaining

$$
\int_{B}\left(\partial_{x} f\right)\left(\partial_{x} \phi\right) d x \wedge d y=-\int_{B_{0}}\left(\partial_{x} \partial_{x} f\right) \phi d x \wedge d y .
$$

Combining with the analogous contribution for $\partial_{y} f$, we conclude that $f$ is weakly harmonic on $B$ if $f$ is harmonic on $B_{0}$.

\subsection{Continuity of Equivariant Connections}

Equivariant connections on $P_{X} \rightarrow X$, even those that are not Yang-Mills, have some regularity if they have finite action. In this section, we prove

Proposition 4.4. Let $r=-1(\bmod 4)$ and $t=-1(\bmod 4)$ with $n_{+} \equiv|r| \neq 1$ and $n_{-} \equiv|t| \neq 1$. A finite-action reduced $(r, t)$ connection corresponds to an equivariant connection on $P_{X} \rightarrow X$ that extends to a continuous connection on the quadrupole bundle $P_{\left(n_{+}, n_{-}\right)} \rightarrow S^{4}$.

This proposition has similarities with results announced by Parker [P1]. We shall prove Proposition 4.4 by showing that the connection form relative to the local section $\delta$ (of Lemma 2.3) extends continuously from $X$ to $S^{4}$.

Lemma 4.5. On $E \times N \subset Y$,

$$
\begin{aligned}
A_{\delta}=\delta^{*} \omega= & -\beta^{1} \otimes a_{1}\left[\cos \left(2 r y_{3}\right) l_{1}-\sin \left(2 r y_{3}\right) l_{2}\right] \\
& -\beta^{2} \otimes a_{2}\left[\sin \left(2 r y_{3}\right) l_{1}+\cos \left(2 r y_{3}\right) l_{2}\right] \\
& -\beta^{3} \otimes a_{3} l_{3}+d y_{3} \otimes 2 r l_{3} .
\end{aligned}
$$

Proof. Any two sections are related by a bundle automorphism (gauge transformation). By inspection of Eqs. (2.13) and (2.17), the gauge transformation that relates $\kappa$ and $\delta$ is the map $\varphi$ of (2.15). The connection forms are now related by the famous formula

$$
A_{\delta}=\varphi^{-1} A_{\kappa} \varphi+\varphi^{-1} d \varphi .
$$

Noting that $\varphi\left(y_{1}, y_{2}, y_{3}\right)=\exp \left(2 r y_{3} l_{3}\right)$, and $d \varphi=\exp \left(2 r y_{3} l_{3}\right) d y_{3} \otimes 2 r l_{3}$, Eq. (4.5) follows from straightforward algebra.

Proof of Proposition 4.4. Let $b=\left(b_{1}, b_{2}, b_{3}\right)$ be a smooth reduced $(r, t)$ connection satisfying the conditions of Lemma 2.6, and such that for all $\theta$ in the subinterval $E=(0, \pi / 6) \subset I$, we have $b_{1}(\theta)=b_{2}(\theta)=0$ and $b_{3}(\theta)=r$. By Lemma 2.6, $b$ corresponds to an equivariant connection on $P_{X} \rightarrow X$ that extends to a smooth connection on $P_{\left(n_{+}, n_{-}\right)} \rightarrow S^{4}$. By Lemma 4.5, the connection form $B_{\delta}$ corresponding to $b$ equals

$$
B_{\delta}=-\beta^{3} \otimes r l_{3}+d y_{3} \otimes 2 r l_{3}
$$

on $E \times N \subset Y . B_{\delta}$ passes to the quotient to give a one-form $\tilde{B}_{\delta}$ on $U \cap X \subset S^{4}$, and this one-form extends to a smooth one-form on $U$. We recall that $U$ is a neighborhood of $Q_{0}$ in $S^{4}$. 
We shall show that the one-form $C \equiv A_{\delta}-B_{\delta}$ has a well-defined limit at $\theta=0$ (namely $C=0$ ), from which it follows that $A_{\delta}=B_{\delta}+C$ can be continuously extended. By (4.5) and (4.7) we have

$$
\begin{aligned}
C= & -\beta^{1} \otimes a_{1}\left[\cos \left(2 r y_{3}\right) l_{1}-\sin \left(2 r y_{3}\right) l_{2}\right] \\
& -\beta^{2} \otimes a_{2}\left[\sin \left(2 r y_{3}\right) l_{1}+\cos \left(2 r y_{3}\right) l_{2}\right] \\
& -\beta^{3} \otimes\left(a_{3}-r\right) l_{3} \\
= & \alpha^{1} \otimes C_{1}+\alpha^{2} \otimes C_{2}+\alpha^{3} \otimes C_{3},
\end{aligned}
$$

where we have further decomposed $C$ relative to the orthonormal basis $\left\{\alpha^{i}\right\}$ of $T^{*} Y$. The components are given by

$$
C_{1}=-\frac{a_{1}}{f_{1}} v_{1}, \quad C_{2}=-\frac{a_{2}}{f_{2}} v_{2}, \quad C_{3}=-\frac{a_{3}-r}{f_{3}} v_{3},
$$

where

$$
\begin{aligned}
& v_{1} \equiv\left[\cos \left(2 r y_{3}\right) l_{1}-\sin \left(2 r y_{3}\right) l_{2}\right], \\
& v_{2} \equiv\left[\sin \left(2 r y_{3}\right) l_{1}+\cos \left(2 r y_{3}\right) l_{2}\right], \\
& v_{3} \equiv l_{3}
\end{aligned}
$$

are $\operatorname{Lie}(H)$-valued functions that do not depend on $\theta$.

It is clear that $\lim _{\theta \rightarrow 0}\left|C_{1,2}\right|=0$. To show that

$$
\lim _{\theta \rightarrow 0}\left|C_{3}\right|=0
$$

we use Lemma 3.5, which states that $\left|a_{3}(\theta)-r\right| \leq \theta \sqrt{K_{3}(\theta)}$, with $\lim _{\theta \rightarrow 0} K_{3}(\theta)=0$, from which Eq. (4.11) follows. We conclude that $\lim _{\theta \rightarrow 0} C=0$, where we are using the Riemannian metric $s^{*} g$, for which $\alpha^{i}$ are orthonormal. Passing to the quotient, $C$ defines a one-form $\tilde{C}$ on $U \cap X$ that extends to a continuous one-form on $U$.

\subsection{Smoothness of Equivariant YM Connections}

In this section, we shall prove Theorem 4.1 by showing that the conditions of Theorem 4.2 are satisfied. We start by proving

Proposition 4.6. The one-form $C=A_{\delta}-B_{\delta}$ belongs to the Sobolev space $W_{1}^{2}(E \times N)$.

To compute the Sobolev norm of a $\operatorname{Lie}(H)$-valued differential form, we evaluate $\nabla C$, where $\nabla$ is the covariant derivative corresponding to the Riemannian connection on the tangent bundle $T S^{4}$, and the flat connection on $P_{Y}$ induced by the local trivialization $\theta$.

Using the orthonormal basis $\alpha^{i}$ of the cotangent bundle, the covariant derivative is expressed by the matrix $\omega_{j}^{i}$ of connection forms ${ }^{7}$ on $S^{4}$.

\footnotetext{
$7 \omega_{j}^{i}$ should not be confused with $\omega$, the $\operatorname{Lie}(H)$-valued connection form on $P_{Y} \rightarrow Y$
} 
Lemma 4.7. The connection one-forms of the torsionless Riemannian connection on TY are given by

$$
\begin{array}{lll}
\omega_{1}^{0}=-\frac{f_{1}^{\prime}}{f_{1}} \alpha^{1}, & \omega_{2}^{0}=-\frac{f_{2}^{\prime}}{f_{2}} \alpha^{2}, & \omega_{3}^{0}=-\frac{f_{3}^{\prime}}{f_{3}} \alpha^{3}, \\
\omega_{2}^{1}=-\frac{1}{f_{3}} \alpha^{3}, & \omega_{3}^{2}=-\frac{1}{f_{1}} \alpha^{1}, & \omega_{1}^{3}=+\frac{1}{f_{2}} \alpha^{2} .
\end{array}
$$

with $\omega_{j}^{i}=-\omega_{i}^{j}$.

Proof. We check that the Cartan structure equations [CDD, Chap. V]

$$
d \alpha^{i}+\sum_{j} \omega_{j}^{i} \wedge \alpha^{j}=0,
$$

are satisfied. Starting with $d \alpha^{0}=d d \theta=0$, the $i=0$ equation holds trivially since $\alpha^{j} \wedge \alpha^{j}=0$. For the remaining equations, e.g. $i=1$,

$$
\begin{aligned}
d \alpha^{1} & =d f_{1} \wedge \beta^{1}+f_{1} d \beta^{1} \\
& =\frac{f_{1}^{\prime}}{f_{1}} \alpha^{0} \wedge \alpha^{1}-\frac{f_{1}}{f_{2} f_{3}} \alpha^{2} \wedge \alpha^{3},
\end{aligned}
$$

where we used the Maurer-Cartan equation. Now (4.12) holds because $f_{1}-f_{2}+$ $f_{3}=0$.

We need one more lemma before proving Proposition 4.6.

Lemma 4.8. Let a be a smooth reduced $(r, t)$ connection with finite action $S(a)=$ $\pi^{2} M$. Then

$$
\int_{0}^{\pi / 6} \frac{\left(a_{3}-r\right)^{2}}{\theta^{3}} d \theta \leq 3 M .
$$

Proof. Let $q=a_{3}-r$, so $q(0)=0$. By Lemma 3.5, we have $|q(\theta)| \leq \sqrt{M} \theta$. Defining $g=q / \theta$, we have $g^{2} \leq M$,

$$
\begin{aligned}
2 S / \pi^{2} \geq 2 \int_{0}^{\pi / 6} d \theta\left(a_{3}^{\prime}\right)^{2} G_{3} & \geq \int_{0}^{\pi / 6} d \theta\left(q^{\prime}\right)^{2} / \theta \\
& =\int_{0}^{\pi / 6} d \theta\left\{\theta(q / \theta)^{\prime}+q / \theta\right\}^{2} / \theta \\
& \geq \int_{0}^{\pi / 6} d \theta\left\{2 g g^{\prime}+q^{2} / \theta^{3}\right\} \\
& =g^{2}(\pi / 6)-g^{2}(0)+\int_{0}^{\pi / 6} d \theta\left(q^{2} / \theta^{3}\right) \\
& \geq-M+\int_{0}^{\pi / 6} d \theta\left(q^{2} / \theta^{3}\right) .
\end{aligned}
$$

Adding $M$ to both sides of the inequality completes the proof. 
Proof of Proposition 4.6. Since $C$ passes to a form on the quotient that extends continuously to $S^{4},|C|$ is bounded and hence square integrable on $E \times N$. We now need to show that $\nabla C \in L^{2}$. To compute the components of $\nabla C$ with respect to the orthonormal basis $\alpha^{k} \otimes \alpha^{i}$ of $T^{*} \otimes T^{*}$, we use

$$
\begin{aligned}
\nabla C & =\sum_{k}\left(\alpha^{k} \otimes d C_{k}+\nabla \alpha^{k} \otimes C_{k}\right) \\
& =\sum_{k, i}\left(\alpha^{k} \otimes \alpha^{i} \otimes \mathscr{L}_{e_{k}} C_{i}-\omega_{i}^{k} \otimes \alpha^{i} \otimes C_{k}\right) \\
& =\sum_{k, i}(\nabla C)_{k i} \alpha^{k} \otimes \alpha^{i}
\end{aligned}
$$

where $\mathscr{B}$ is the Lie derivative, and $\omega_{i}^{k}$ are the Riemannian connection forms of Lemma 4.7. A straightforward computation gives ${ }^{8}$

$$
\begin{aligned}
& (\nabla C)_{00}=0 \\
& (\nabla C)_{01}=\mathscr{L}_{e_{0}} C_{1}=C_{1}^{\prime}=\left(\frac{a_{1}}{f_{1}}\right)^{\prime} v_{1} \\
& (\nabla C)_{02}=C_{2}^{\prime}=\left(\frac{a_{2}}{f_{2}}\right)^{\prime} v_{2} \\
& (\nabla C)_{03}=C_{3}^{\prime}=\left(\frac{a_{3}-r}{f_{3}}\right)^{\prime} v_{3} \\
& (\nabla C)_{10}=\frac{-f_{1}^{\prime}}{f_{1}} C_{1}=\frac{f_{1}^{\prime} a_{1}}{f_{1}^{2}} v_{1}, \\
& (\nabla C)_{11}=\frac{1}{f_{1}} \mathscr{L}_{l_{1}} C_{1}=-\frac{a_{1}}{f_{1}^{2}} \mathscr{L}_{l_{1}} v_{1}, \\
& (\nabla C)_{12}=\frac{1}{f_{1}}\left(\mathscr{L}_{l_{1}} C_{2}-C_{3}\right)=\frac{1}{f_{1}}\left(\frac{-a_{2}}{f_{2}} \mathscr{L}_{l_{1}} v_{2}+\frac{a_{3}-r}{f_{3}} v_{3}\right) \\
& (\nabla C)_{13}=\frac{1}{f_{1}}\left(\mathscr{L}_{l_{1}} C_{3}+C_{2}\right)=\frac{1}{f_{1}}\left(\frac{-\left(a_{3}-r\right)}{f_{3}} \mathscr{L}_{l_{1}} v_{3}+\frac{a_{2}}{f_{2}} v_{2}\right) \\
& (\nabla C)_{20}=\frac{-f_{2}^{\prime}}{f_{2}} C_{2}=\frac{f_{2}^{\prime} a_{2}}{f_{2}^{2}} v_{2}, \\
& (\nabla C)_{21}=\frac{1}{f_{2}}\left(\mathscr{L}_{l_{2}} C_{1}-C_{3}\right)=\frac{1}{f_{2}}\left(\frac{-a_{1}}{f_{1}} \mathscr{L}_{l_{2}} v_{1}+\frac{a_{3}-r}{f_{3}} v_{3}\right) \\
& (\nabla C)_{22}=\frac{1}{f_{2}} \mathscr{L}_{l_{2}} C_{2}=-\frac{a_{2}}{f_{2}^{2}} \mathscr{L}_{l_{2}} v_{2}, \\
& (\nabla C)_{23}=\frac{1}{f_{2}}\left(\mathscr{L}_{l_{2}} C_{3}+C_{1}\right)=\frac{1}{f_{2}}\left(\frac{-\left(a_{3}-3\right)}{f_{3}} \mathscr{L}_{l_{2}} v_{3}-\frac{a_{1}}{f_{1}} v_{1}\right) \\
& (\nabla) \\
& (\nabla) \\
& (\nabla) \\
& (\nabla) \\
& (\nabla)
\end{aligned}
$$

\footnotetext{
${ }^{8}$ Since we have identified $G=H, l_{i}$ denotes both vector fields on $G$, as in $\mathscr{L}_{l_{i}}$, and also the basis
} of $\operatorname{Lie}(H)$, as in $v_{3}=l_{3}$. The meaning should be clear from context 


$$
\begin{aligned}
& (\nabla C)_{30}=\frac{-f_{3}^{\prime}}{f_{3}} C_{3}=\frac{f_{3}^{\prime}\left(a_{3}-r\right)}{f_{3}^{2}} v_{3} \\
& (\nabla C)_{31}=\frac{1}{f_{3}}\left(\mathscr{L}_{l_{3}} C_{1}-C_{2}\right)=\frac{1}{f_{3}}\left(\frac{-a_{1}}{f_{1}} \mathscr{L}_{l_{3}} v_{1}+\frac{a_{2}}{f_{2}} v_{2}\right) \\
& (\nabla C)_{32}=\frac{1}{f_{3}}\left(\mathscr{L}_{l_{3}} C_{2}+C_{1}\right)=\frac{1}{f_{3}}\left(\frac{-a_{2}}{f_{2}} \mathscr{L}_{l_{3}} v_{2}+\frac{a_{1}}{f_{1}} v_{1}\right) \\
& (\nabla C)_{33}=\frac{1}{f_{3}} \mathscr{L}_{l_{3}} C_{3}=\frac{-\left(a_{3}-r\right)}{f_{3}^{2}} \mathscr{L}_{l_{3}} v_{3} .
\end{aligned}
$$

For each pair of indices $k, i$ we must show that

$$
T_{k, i} \equiv \int_{E \times N}\left|(\nabla C)_{k i}\right|^{2} \eta
$$

is finite, where $\eta=f_{1} f_{2} f_{3} d \theta \wedge \beta^{1} \wedge \beta^{2} \wedge \beta^{3}$ is the volume element on $Y$. The $\operatorname{Lie}(H)$ valued functions $v_{i}$ and $\mathscr{L}_{l_{i}} v_{k}$ are bounded and independent of $\theta$, so we need only look at the coefficients. Using the fact that $f_{3}=O(\theta)$ and $f_{1}$ and $f_{2}$ are $O(1)$, we easily bound all the $T_{k, i}$ 's, by a multiple of

$$
\int\left[\frac{\left(a_{1}\right)^{2}}{\theta}+\left(a_{1}^{\prime}\right)^{2} \theta+\frac{\left(a_{2}\right)^{2}}{\theta}+\left(a_{2}^{\prime}\right)^{2} \theta+\left(a_{3}^{\prime}\right)^{2} / \theta+\left(a_{3}-r\right)^{2} / \theta^{3}\right] d \theta \text {. }
$$

The integral of the first five terms is finite by Lemma 3.7, and the integral of the last term is finite by Lemma 4.8 .

We are now ready to prove the regularity theorem.

Proof of Theorem 4.1. Since $B_{\delta}$ is smooth and $C=A_{\delta}-B_{\delta}$ belongs to $W_{1}^{2}(E \times N)$ by Proposition 4.6, $A_{\delta} \in W_{1}^{2}(E \times N)$. The one-form $A_{\delta}$ on $E \times N$ passes to the quotient, defining a one-form $\tilde{A}_{\delta}$ on $U \cap X \subset S^{4}$, and $\tilde{A}_{\delta}$ belongs to $W_{1}^{2}(U \cap X)$. The singular set has codimension two, and using Fubini's theorem, as in the proof of Proposition 4.3, we conclude that $\tilde{A}_{\delta} \in W_{1}^{2}(U)$.

Since the reduced connection $a$ is a solution of the reduced Yang-Mills equations, $A_{\kappa}$ is a solution of the Yang-Mills equation on $Y$. The YM equations are gaugeinvariant, so $A_{\delta}$ is a solution on $E \times N$. Passing to the quotient, $\tilde{A}_{\delta}$ is a solution of the YM equations on $U \cap X \subset S^{4}$. Since the singular set has codimension two, we use Fubini's theorem again, as in the proof of Proposition 4.3, to conclude that $\tilde{A}_{\delta}$ is a weak solution (in the sense of [Sed, Eq. (4.1)]) of the YM equations on all of $U$.

Now by Theorem 4.2, on some neighborhood $\tilde{U}$ of $Q_{0}$ in $S^{4}$, there is a gauge transformation $\phi \in W_{2}^{2}(\tilde{U})$ that takes $\widetilde{A}_{\delta}$ to a connection form $A$ that is a smooth solution of the YM equations on $\tilde{U}$. This gauge transformation solves the equation

$$
d \phi=\phi A-\tilde{A}_{\delta} \phi .
$$

Now $A$ is smooth on $\tilde{U}$, and $\tilde{A}_{\delta}$ is continuous on $\tilde{U}$ by Proposition 4.4, so we conclude that $\phi$ is actually continuously differentiable on $\tilde{U}$. Moreover, since $\tilde{A}_{\kappa}$ and hence $\tilde{A}_{\delta}$ are smooth on $\tilde{U} \cap X$, the gauge transformation $\phi$ is in addition smooth on $\tilde{U} \cap X$. (See e.g. [JT], Lemma 6.2 for details on this type of argument.)

By covering the singular orbits with patches, and repeating this argument, we obtain the existence of a smooth YM connection on a bundle that is $C^{1}$ isomorphic, hence $C^{\infty}$ isomorphic ([JT], Theorem 4.4) to the quadrupole bundle. 
Now we can prove our main theorem.

Proof of Theorem 1.1. For any two odd integer $n_{ \pm}>1$ we can choose $r= \pm n_{+}$ such that $r=-1(\bmod 4)$ and $t= \pm n_{-}$such that $t=-1(\bmod 4)$. By Theorem 3.1 there exists a smooth reduced $(r, t)$ connection that is a non-self-dual solution of the reduced Yang-Mills equations. By Theorem 4.1 this corresponds to a smooth (non-self-dual) Yang-Mills connection on the quadrupole bundle $P_{\left(n_{+}, n_{-}\right)} \rightarrow S^{4}$.

Acknowledgements. As previously mentioned, much of Sect. 2 is due to Bor and Montgomery [BoMo], who applied the general theory of Urakawa [Ur] to quadrupole bundles. We have also benefitted from discussions and/or correspondence with Percy Deift, Lesley Sibner, Robert Sibner, Barry Simon, Cliff Taubes, and particularly Jalal Shatah.

\section{References}

[Ad] Adams, R.A.: Sobolev spaces. New York: Academic Press 1975

[ADHM] Atiyah, M.F., Drinfeld, V.G., Hitchin, N.J., Manin, Y.I.: Construction of instantons. Phys. Lett. A 65, 185 (1978)

[AJ] Atiyah, M.F., Jones, J.D.S.: Topological aspects of Yang-Mills theory. Commun. Math. Phys. 61, 97 (1978)

[ASSS] Avron, J.E., Sadun, L., Segert, J., Simon, B.: Chern numbers, quaternions, and Berry's phases in fermi systems. Commun. Math. Phys. 124, 595 (1989)

[Au] Aubin, T.: Nonlinear analysis on manifolds. Monge-Ampere equations. Berlin, Heidelberg, New York: Springer 1982

[Ber] Berger, M.S.: Nonlinearity and functional analysis. New York: Academic Press 1977

[BoMo] Bor, G., Montgomery, R.: SO(3) invariant Yang-Mills fields which are not self-dual. Proceedings of the MSI Workshop on Hamiltonian Systems, Transformation Groups, and Spectral Transform Methods, held in Montreal, Canada, Oct. 1989

[BL] Bourguignon, J.P., Lawson, H.B.: Stability and isolation phenomena for Yang-Mills equations. Commun. Math. Phys. 79, 189 (1982)

[BLS] Bourguignon, J.P., Lawson, H.B., Simons, J.: Stability and gap phenomena for Yang-Mills fields. Proc. Natl. Acad. Sci. USA 76, 1550 (1979)

[BPST] Belavin, A.A., Polyakov, A.M., Schwartz, A.S., Tyupkin, Yu.: Pseudo-particle solutions of the Yang-Mills equations. Phys. Lett. B 59, 85 (1975)

[CDD] Choquet-Bruhat, Y., DeWitt-Morette, C., Dillard-Bleick, M.: Analysis, manifolds, and physics. Amsterdam: North-Holland 1982

[FHP1] Forgacs, P., Horvath, Z., Palla, L.: An exact fractionally charged self-dual solution. Phys. Rev. Lett. 46, 392 (1981)

[FHP2] Forgacs, P., Horvath, Z., Palla, L.: One can have noninteger topological charge. Z. Phys. C - Particles and Fields 12, 359-360 (1982)

[FU] Freed, D.S., Uhlenbeck, K.K.: Instantons and four-manifolds. Berlin, Heidelberg, New York: Springer 1984

[I] Itoh, M.: Invariant connections and Yang-Mills solutions. Trans. Am. Math. Soc. 267, $229(1981)$

[JT] Jaffe, A., Taubes, C.: Vortices and monopoles. Boston: Birkhäuser 1980

[LU] Ladyzhenskaya, O., Ural'tseva, N.: Linear and quasilinear elliptic partial differential equations. New York: Academic Press 1968

[Ma1] Manin, Yu.: New exact solutions and cohomology analysis of ordinary and supersymmetric Yang-Mills equations. Proc. Steklov Inst. of Math. 165, 107 (1984)

[Ma2] Manin, Yu.: Gauge field theory and complex geometry. Berlin, Heidelberg, New York: Springer 1988

[P1] Parker, T.: Unstable Yang-Mills fields. Preprint 1989

[P2] Parker, T.: Non-minimal Yang-Mills fields and dynamics. Invent. Math. (in press)

[Pal] Palais, R.S.: The principle of symmetric criticality. Commun. Math. Phys. 69, 19 (1979)

[RS] Reed, M., Simon, B.: Methods of modern mathematical physics, Vol. I, II. New York: Academic Press 1980 
[SaU] Sacks, J., Uhlenbeck, K.: On the existence of minimal immersions of 2-spheres. Ann. Math. 113(2), 1-24 (1982)

[Sed] Sedlacek, S.: A direct method for minimizing the Yang-Mills functional over 4-manifolds. Commun. Math. Phys. 86, 515-527 (1982)

[SS1] Sadun, L., Segert, J.: Chern numbers for fermionic quadrupole systems. J. Phys. A 22, L 111 (1989)

[SS2] Sadun, L., Segert, J.: Non-self-dual Yang-Mills connections with nonzero Chern number. Bull. Am. Math. Soc. 24, 163-170 (1991)

[SS3] Sadun, L., Segert, J.: Stationary points of the Yang-Mills action. Commun. Pure Appl. Math. (in press)

[SiSi1] Sibner, L.M., Sibner, R.J.: Singular Sobolev connections with holonomy. Bull. Am. Math. Soc. 19, 471-473 (1988)

[SiSi2] Sibner, L.M., Sibner, R.J.: Classification of singular Sobolev connections by their holonomy. Commun. Math. Phys. (to appear)

[SSU] Sibner, L.M., Sibner, R.J., Uhlenbeck, K.: Solutions to Yang-Mills equations which are not self-dual. Proc. Natl. Acad. Sci USA 86, 860-863 (1989)

[T1] Taubes, C.H.: Stability in Yang-Mills theories. Commun. Math. Phys. 91, 235 (1983)

[T2] Taubes, C.H.: On the equivalence of the first and second Order equations for gauge theories. Commun. Math. Phys. 75, 207 (1980)

[Uh1] Uhlenbeck, K.: Removable singularities in Yang-Mills fields. Commun. Math. Phys. 83, 11-29 (1982)

[Uh2] Uhlenbeck, K.: Connections with $L^{p}$ bounds on curvature. Commun. Math. Phys. 83, 31-42 (1982)

[Uh3] Uhlenbeck, K.: Variational problems for gauge fields. In Seminar on Differential Geometry. Yau, S.-T. (ed.). Princeton: Princeton University Press 1982

[Ur] Urakawa, H.: Equivariant theory of Yang-Mills connections over Riemannian manifolds of cohomogeneity one. Indiana Univ. Math. J. 37, 753 (1988)

Communicated by T. Spencer

Note Added in Proof. This paper was submitted to Ann. Math. in September 1990, and upon suggestion of the editors, was subsequently transferred without revision to Commun. Math. Phys. We have in the meantime received the following papers:

[Bo] Bor, G.: Yang-Mills fields which are not self-dual. Commun. Math. Phys. 145, 393-410 (1992)

[P3] Parker, T.H.: A Morse Theory for Equivariant Yang-Mills. Duke Math. J. (in press)

[W] Wang, H.-Y.: The existence of non-minimal solutions to the Yang-Mills equation with group $S U(2)$ on $S^{2} \times S^{2}$ and $S^{1} \times S^{3}$. J. Diff. Geom. 34, 701-767 (1991) 
